\title{
Convergence Rates for Projective Splitting
}

\author{
Patrick R. Johnstone* Jonathan Eckstein*
}

August 10, 2018

\begin{abstract}
Projective splitting is a family of methods for solving inclusions involving sums of maximal monotone operators. First introduced by Eckstein and Svaiter in 2008, these methods have enjoyed significant innovation in recent years, becoming one of the most flexible operator splitting frameworks available. While weak convergence of the iterates to a solution has been established, there have been few attempts to study convergence rates of projective splitting. The purpose of this paper is to do so under various assumptions. To this end, there are three main contributions. First, in the context of convex optimization, we establish an $O(1 / k)$ ergodic function convergence rate. Second, for strongly monotone inclusions, strong convergence is established as well as an ergodic $O(1 / \sqrt{k})$ convergence rate for the distance of the iterates to the solution. Finally, for inclusions featuring strong monotonicity and cocoercivity, linear convergence is established.
\end{abstract}

\section{Introduction}

For a real Hilbert space $\mathcal{H}$, consider the problem of finding $z \in \mathcal{H}$ such that

$$
0 \in \sum_{i=1}^{n} T_{i} z
$$

where $T_{i}: \mathcal{H} \rightarrow 2^{\mathcal{H}}$ are maximal monotone operators and additionally there exists a subset $\mathcal{I}_{\mathrm{F}} \subseteq\{1, \ldots, n\}$ such that for all $i \in \mathcal{I}_{\mathrm{F}}$ the operator $T_{i}$ is Lipschitz continuous. An important instance of this problem is

$$
\min _{z \in \mathcal{H}} F(z), \quad \text { where } \quad F(z)=\sum_{i=1}^{n} f_{i}(z)
$$

and every $f_{i}: \mathcal{H} \rightarrow(-\infty,+\infty]$ is closed, proper, and convex, with some subset of the functions also being Fréchet differentiable with Lipschitz-continuous gradients. Under appropriate constraint qualifications, (11) and (21) are equivalent. Problem (21) arises in a host

${ }^{*}$ Department of Management Science and Information Systems, Rutgers Business School Newark and New Brunswick, Rutgers University 
of applications such as machine learning, signal and image processing, inverse problems, and computer vision; see [3, 5, 6] for some examples.

A relatively recently proposed class of operator splitting algorithms which can solve (11), among other problems, is projective splitting. It originated with [10] and was then generalized to more than two operators in [11]. The related algorithm in [1] introduced a technique for handling compositions of linear and monotone operators, and [4] proposed an extension to "block-iterative" and asynchronous operation - block-iterative operation meaning that only a subset of the operators making up the problem need to be considered at each iteration (this approach may be called "incremental" in the optimization literature). A restricted and simplified version of this framework appears in [8]. The recent work [15] incorporated forward steps into the projective splitting framework for any Lipschitz continuous operators and introduced backtracking and adaptive stepsize rules.

In general, projective splitting offers unprecedented levels of flexibility compared with previous operator splitting algorithms (e.g. [20, 17, 24, 7]). The framework can be applied to arbitary sums of maximal monotone operators, the stepsizes can vary by operator and by iteration, compositions with linear operators can be handled, and block-iterative asynchronous implementations have been demonstrated.

In previous works on projective splitting, the main theoretical goal was to establish the weak convergence of the iterates to a solution of the monotone inclusion under study (either a special case or a generalization of (1)). This goal was achieved using Fejér-monotonicity arguments in coordination with the unique properties of projective splitting. The question of convergence rates has not been addressed, with the sole exception of [19], which considers a different type of convergence rate than those investigated here; we discuss the differences between our analysis and that of [19] in more detail below.

\section{Contributions}

To this end, there are four main novel contributions in this paper.

1. For (2), we establish an ergodic $\mathrm{O}(1 / k)$ function value convergence rate for iterates generated by projective splitting.

2. When one of the operators in (11) is strongly monotone, we establish strong convergence, rather than weak, in the general Hilbert space setting, without using the Haugazeau [13] modification employed to obtain general strong convergence in [4]. Furthermore, we derive an ergodic $\mathrm{O}(1 / \sqrt{k})$ convergence rate for the distance of the iterates to the unique solution of (1).

3. If additionally $T_{1}, \ldots, T_{n-1}$ are cocoercive, we establish linear convergence to 0 of the distance of the iterates to the unique solution.

4. We discuss the special cases of projective splitting when $n=1$. Interestingly, projective splitting reduces to one of two well-known algorithms in this case depending on whether forward or backward steps are used. This observation has implications for the convergence rate analysis.

The primary engine of the analysis is a new summability lemma (Lemma 8 below) in which important quantities of the algorithm are shown to be summable. This summability 
is directly exploited in the ergodic function value rate analysis in Section 6. In Section 8, the same lemma is used to show linear convergence when strong monotonicity and cocoercivity are present. With only strong monotonicity present, we also obtain strong convergence and rates using a novel analysis in Section 7.

Our convergence rates apply directly to the variants of projective splitting discussed in [4, 10, 15]. The papers [8, 11] use a slightly different separating hyperplane formulation than ours but the difference is easy to resolve. However, our analysis does not allow for the asynchrony or block-iterative effects developed in [4, 8, 15]. In particular, at each iteration we assume that every operator is processed and that the computations use the most up-todate information. Developing a convergence rate analysis which extends to asynchronous and block-iterative operation in a satisfactory manner is a matter for future work.

In [4, 8, 15], projective splitting was extended to handle the composition of each $T_{i}$ with a linear operator. While it is possible to extend all of our convergence rate results to allow for this generalization under appropriate conditions, for the sake of readability we will not do so here.

In Section 9 we consider the case $n=1$. In this case, we show that projective splitting reduces to the proximal point method [22] if one uses backward steps, or to a special case of the extragradient method (with no constraint) [16, 21] when one uses forward steps. Since projective splitting includes the proximal point method as a special case, the $\mathrm{O}(1 / k)$ function value convergence rate derived in Section 6 cannot be improved, since this is the best rate available for the proximal point method, as established in [12].

The specific outline for the paper is as follows. Immediately below, we discuss in more detail the convergence rate analysis for projective splitting conducted in [19] and how it differs from our analysis. Section 2 presents notation, basic mathematical results, and assumptions. Section 3 introduces the projective splitting framework under study, along with some associated assumptions. Section 4 recalls some important lemmas from 15 . Section 5 proves some new lemmas necessary for convergence rate results, including the key summability lemma (Lemma 8). Section 6 derives the ergodic $\mathrm{O}(1 / k)$ function value convergence rate for (2). Section 7 derives strong convergence and convergence rates under strong monotonicity. Section 8 establishes linear convergence under strong monotonicity and cocoercivity. Finally, Section 9 discusses special cases of projective splitting when $n=1$.

\section{Comparison with 19]}

To the best of our knowledge, the only works attempting to quantify convergence rates of projective splitting are [18] and [19], two works by the same author. The analysis in [18] concerns a dual application of projective splitting and its convergence rate results are similar to those in [19]. In these works, convergence rates are not defined in the more customary way they are in this paper - either in terms of the distance to the solution or the gap between current function values and the optimal value of (2). Instead in [19] they are defined in terms of an approximate solution criterion for the monotone inclusion under study, specifically (11) with $n=2$. Without any enlargement being applied to the operators, the approximate solution condition is as follows: a point $(x, y) \in \mathcal{H}^{2}$ is said to be an $\epsilon$-approximate solution of (1) with $n=2$ if there exists $(a, b) \in \mathcal{H}^{2}$ s.t. $a \in T_{1} x$, $b \in T_{2} y$ and $\max \{\|a+b\|,\|x-y\|\} \leq \epsilon$. If this condition holds with $\epsilon=0$, then $x=y$ is a solution to (11). The iteration complexity of a method is then defined in terms of the 
number iterations required to produce a point $\left(x^{k}, y^{k}\right)$ which is a $\epsilon$-approximate solution in this sense.

We stress that this notion of iteration complexity/convergence rate is different to what we use here. Instead, we directly analyze the distance of the points generated by the algorithm to a solution of (11), that is, we study the behavior of $\left\|z^{k}-z^{*}\right\|$, where $z^{*}$ solves (1). Or for the special case of (2), we consider the convergence rate of $F\left(z^{k}\right)-F^{*}$ to zero, where $F^{*}$ is the optimal value.

\section{Mathematical Preliminaries}

\subsection{Notation, Terminology, and Basic Lemmas}

Throughout this paper we will use the standard convention that a sum over an empty set of indices, such as $\sum_{i=1}^{n-1} a_{i}$ with $n=1$, is taken to be 0 . A single-valued operator $A: \mathcal{H} \rightarrow \mathcal{H}$ is called cocoercive if, for some $\Gamma>0$,

$$
(\forall u, v \in \mathcal{H}) \quad\langle u-v, A(u)-A(v)\rangle \geq \Gamma^{-1}\|A(u)-A(v)\|_{2}^{2} .
$$

Every cocoercive operator is $\Gamma$-Lipschitz continuous, but not vice versa. For any maximal monotone operator $A: \mathcal{H} \rightarrow 2^{\mathcal{H}}$ and scalar $\rho>0$ we will use the notation $\operatorname{prox}_{\rho A}=(I+\rho A)^{-1}$ to denote the proximal operator, also known as the backward or implicit step with respect to $A$. In particular,

$$
x=\operatorname{prox}_{\rho A}(a) \quad \Longrightarrow \quad \exists y \in A x: x+\rho y=a,
$$

and the $x$ and $y$ satisfying this relation are unique. Furthermore, $\operatorname{prox}_{\rho A}$ is defined everywhere and $\operatorname{range}\left(\operatorname{prox}_{A}\right)=\operatorname{dom}(A)$ [2, Proposition 23.2]. In the special case where $A=\partial f$ for some convex, closed, and proper function $f$, the proximal operator may be written as

$$
\operatorname{prox}_{\rho \partial f}=\underset{x}{\arg \min }\left\{\frac{1}{2}\|x-a\|^{2}+\rho f(x)\right\} .
$$

In the optimization context, we will use the notational convention that $\operatorname{prox}_{\rho \partial f}=\operatorname{prox}_{\rho f}$. Finally, we will use the following two standard results:

Lemma 1. For any vectors $v_{1}, \ldots, v_{n} \in \mathcal{H}^{n},\left\|\sum_{i=1}^{n} v_{i}\right\|^{2} \leq n \sum_{i=1}^{n}\left\|v_{i}\right\|^{2}$.

Lemma 2. For any $x, y, z \in \mathcal{H}$

$$
2\langle x-y, x-z\rangle=\|x-y\|^{2}+\|x-z\|^{2}-\|y-z\|^{2} .
$$

We will use a boldface $\mathbf{w}=\left(w_{1}, \ldots, w_{n-1}\right)$ for elements of $\mathcal{H}^{n-1}$.

\subsection{Main Assumptions Regarding Problem (1)}

Define the extended solution set or Kuhn-Tucker set of (1) to be

$$
\mathcal{S}=\left\{\left(z, w_{1}, \ldots, w_{n-1}\right) \in \mathcal{H}^{n} \mid w_{i} \in T_{i} z, i=1, \ldots, n-1,-\sum_{i=1}^{n-1} w_{i} \in T_{n} z\right\} .
$$

Clearly $z \in \mathcal{H}$ solves (11) if and only if there exists $\mathbf{w} \in \mathcal{H}^{n-1}$ such that $(z, \mathbf{w}) \in \mathcal{S}$. Our main assumptions regarding (11) are as follows: 
Assumption 1. $\mathcal{H}$ is a real Hilbert space and problem (1) conforms to the following:

1. For $i=1, \ldots, n$, the operators $T_{i}: \mathcal{H} \rightarrow 2^{\mathcal{H}}$ are monotone.

2. For all $i$ in some subset $\mathcal{I}_{\mathrm{F}} \subseteq\{1, \ldots, n\}$, the operator $T_{i}$ is $L_{i}$-Lipschitz continuous (and thus single-valued) and $\operatorname{dom}\left(T_{i}\right)=\mathcal{H}$.

3. For $i \in \mathcal{I}_{\mathrm{B}} \triangleq\{1, \ldots, n\} \backslash \mathcal{I}_{\mathrm{F}}$, the operator $T_{i}$ is maximal and that the map prox ${ }_{\rho T_{i}}$ : $\mathcal{H} \rightarrow \mathcal{H}$ can be computed to within the error tolerance specified below in Assumption 2 (however, these operators are not precluded from also being Lipschitz continuous).

4. The solution set $\mathcal{S}$ defined in (6) is nonempty.

Proposition 1. [15, Lemma 3] Under Assumption 1, $\mathcal{S}$ from (6) is closed and convex.

\section{The Algorithm}

Projective splitting is a special case of a general seperator-projector method for finding a point in a closed and convex set. At each iteration the method constructs an affine function $\varphi_{k}: \mathcal{H}^{n} \rightarrow \mathbb{R}$ which separates the current point from the target set $\mathcal{S}$ defined in (6). In other words, if $p^{k}$ is the current point in $\mathcal{H}^{n}$ generated by the algorithm, $\varphi_{k}\left(p^{k}\right)>0$, and $\varphi_{k}(p) \leq 0$ for all $p \in \mathcal{S}$. The next point is then the projection of $p^{k}$ onto the hyperplane $\{p:$ $\left.\varphi_{k}(p)=0\right\}$, subject to a relaxation factor $\beta_{k}$. What makes projective splitting an operator splitting method is that the hyperplane is constructed through individual calculations on each operator $T_{i}$, either prox calculations or forward steps.

\subsection{The Hyperplane}

Let $p=(z, \mathbf{w})=\left(z, w_{1}, \ldots, w_{n-1}\right)$ be a generic point in $\mathcal{H}^{n}$. For $\mathcal{H}^{n}$, we adopt the following norm and inner product for some $\gamma>0$ :

$$
\|(z, \mathbf{w})\|^{2}=\gamma\|z\|^{2}+\sum_{i=1}^{n-1}\left\|w_{i}\right\|^{2} \quad\left\langle\left(z^{1}, \mathbf{w}^{1}\right),\left(z^{2}, \mathbf{w}^{2}\right)\right\rangle_{\gamma}=\gamma\left\langle z^{1}, z^{2}\right\rangle+\sum_{i=1}^{n-1}\left\langle w_{i}^{1}, w_{i}^{2}\right\rangle .
$$

Define the following function for all $k \geq 1$ :

$$
\varphi_{k}(p)=\sum_{i=1}^{n-1}\left\langle z-x_{i}^{k}, y_{i}^{k}-w_{i}\right\rangle+\left\langle z-x_{n}^{k}, y_{n}^{k}+\sum_{i=1}^{n-1} w_{i}\right\rangle .
$$

where the $\left(x_{i}^{k}, y_{i}^{k}\right)$ are chosen so that $y_{i}^{k} \in T_{i} x_{i}^{k}$ for $i=1, \ldots, n$. This function is a special case of the separator function used in [4]. The following lemma proves some basic properties of $\varphi_{k} ;$ similar results are in [1, 4, 8] in the case $\gamma=1$.

Lemma 3. [15, Lemma 4] Let $\varphi_{k}$ be defined as in (8). Then:

1. $\varphi_{k}$ is affine on $\mathcal{H}^{n}$. 
2. With respect to inner product (77) defined on $\mathcal{H}^{n}$, the gradient of $\varphi_{k}$ is

$$
\nabla \varphi_{k}=\left(\frac{1}{\gamma}\left(\sum_{i=1}^{n} y_{i}^{k}\right), x_{1}^{k}-x_{n}^{k}, x_{2}^{k}-x_{n}^{k}, \ldots, x_{n-1}^{k}-x_{n}^{k}\right) .
$$

3. Suppose Assumption 11 holds and that $y_{i}^{k} \in T_{i} x_{i}^{k}$ for $i=1, \ldots, n$. Then $\varphi_{k}(p) \leq 0$ for all $p \in \mathcal{S}$ defined in (6).

4. If Assumption 1 holds, $y_{i}^{k} \in T_{i} x_{i}^{k}$, and $\nabla \varphi_{k}=0$, then $\left(x_{n}^{k}, y_{1}^{k}, \ldots, y_{n-1}^{k}\right) \in \mathcal{S}$.

\subsection{Projective Splitting}

Algorithm 1 is the projective splitting framework for which we will derive convergence rates. It is a special case of the framework of [15] without asynchrony or block-iterative features. In particular, we assume at each iteration that the method processes every operator $T_{i}$, using the most up-to-date information possible. The frameworks of [4, 8, 15] also allow for the monotone operators to be composed with linear operators. As mentioned in the introduction, our analysis may be extended to this situation, but for the sake of readibility we will not do so here.

Algorithm 1 is a special case of the separator-projector algorithm applied to finding a point in $\mathcal{S}$ using the affine function $\varphi_{k}$ defined in (8) [15, Lemma 6]. For the variables of Algorithm 1 defined on lines 18 19, define $p^{k}=\left(z^{k}, \mathbf{w}^{k}\right)^{\top}=\left(z^{k}, w_{1}^{k}, \ldots, w_{n-1}^{k}\right)^{\top}$ for all $k \geq 1$. The points $\left(x_{i}^{k}, y_{i}^{k}\right) \in$ gra $T_{i}$ are chosen so that $\varphi_{k}\left(p^{k}\right)$ is sufficiently large to guarantee the weak convergence of $p^{k}$ to a solution [15, Theorem 1]. For $i \in \mathcal{I}_{\mathrm{B}}$, a single prox calculation is required, whereas for $i \in \mathcal{I}_{\mathrm{F}}$, two forward steps are required per iteration.

The algorithm has the following parameters:

- For each $k \geq 1$ and $i=1, \ldots, n$, a positive scalar stepsize $\rho_{i}^{k}$.

- For each $k \geq 1$, an overrelaxation parameter $\beta_{k} \in[\underline{\beta}, \bar{\beta}]$ where $0<\underline{\beta} \leq \bar{\beta}<2$.

- The fixed scalar $\gamma>0$ from (7), which controls the relative emphasis on the primal and dual variables in the projection update in lines 18, 19,

- Sequences of errors $\left\{e_{i}^{k}\right\}_{k \geq 1}$ for $i \in \mathcal{I}_{\mathrm{B}}$, modeling inexact computation of the proximal steps.

To ease the mathematical presentation, we use the following notation in Algorithm 1 and throughout the rest of the paper:

$$
(\forall k \in \mathbb{N}) \quad w_{n}^{k} \triangleq-\sum_{i=1}^{n-1} w_{i}^{k} .
$$

Note that when $n=1$, we have $w_{n}^{k}=0$ by the convention at the start of Section 2.1. 


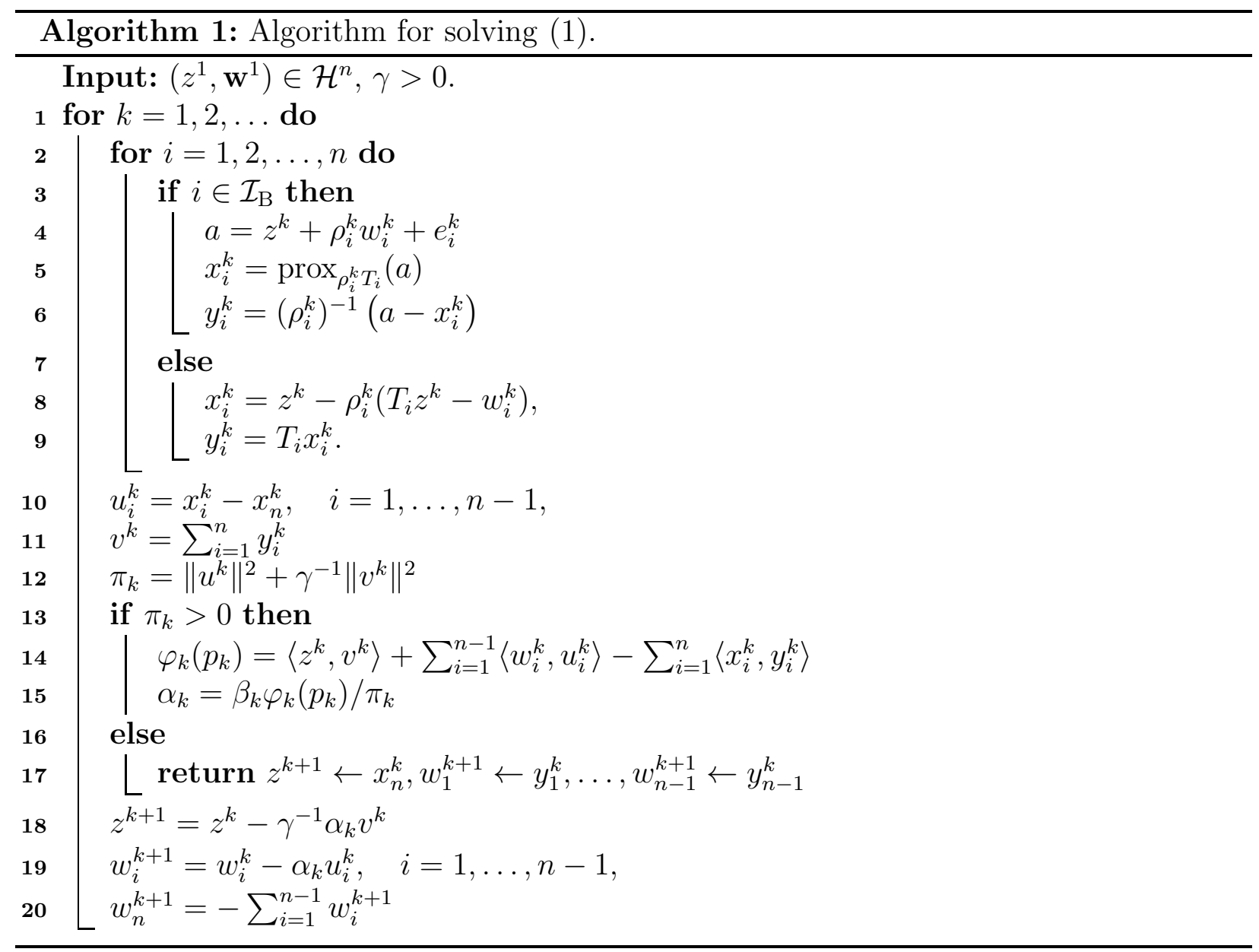

\subsection{Conditions on the Errors and the Stepsizes}

We now state our assumptions regarding the computational errors and stepsizes in Algorithm 1. Assumptions (11) and (12) are taken exactly from [8]. Assumption (13) is new and necessary to derive our convergence rate results. Throughout the rest of the manuscript, let $\bar{K}$ be the iteration where Algorithm 1 terminates via Line 17, with $\bar{K}=\infty$ if the method runs indefinitely.

Assumption 2. For some $\sigma \in[0,1[$ and $\delta \geq 0$, the following hold for all $1 \leq k \leq \bar{K}$ and $i \in \mathcal{I}_{\mathrm{B}}$ :

$$
\begin{aligned}
\left\langle z^{k}-x_{i}^{k}, e_{i}^{k}\right\rangle & \geq-\sigma\left\|z^{k}-x_{i}^{k}\right\|^{2} \\
\left\langle e_{i}^{k}, y_{i}^{k}-w_{i}^{k}\right\rangle & \leq \rho_{i}^{k} \sigma\left\|y_{i}^{k}-w_{i}^{k}\right\|^{2} \\
\left\|e_{i}^{k}\right\|^{2} & \leq \delta\left\|z^{k}-x_{i}^{k}\right\|^{2} .
\end{aligned}
$$

Note that if (13) holds with $\delta<1$, then (11) holds for any $\sigma \in\left[\delta, 1\left[\right.\right.$. For each $i \in \mathcal{I}_{\mathrm{B}}$, we will show in (33) below that the sequence $\left\{\left\|z^{k}-x_{i}^{k}\right\|\right\}$ is square-summable, so an eventual consequence of (13) will be that the corresponding error sequence must be square-summable, that is, $\sum_{k=1}^{\bar{K}}\left\|e_{i}^{k}\right\|^{2}<\infty$. 
Assumption 3. The stepsizes satisfy

$$
\begin{aligned}
& \underline{\rho} \triangleq \min _{i=1, \ldots, n}\left\{\inf _{1 \leq k \leq \bar{K}} \rho_{i}^{k}\right\}>0 \\
& \left(\forall i \in \mathcal{I}_{\mathrm{B}}\right) \quad \bar{\rho}_{i} \triangleq \sup _{1 \leq k \leq \bar{K}} \rho_{i}^{k}<\infty \\
& \left(\forall i \in \mathcal{I}_{\mathrm{F}}\right) \quad \bar{\rho}_{i} \triangleq \sup _{1 \leq k \leq \bar{K}} \rho_{i}^{k}<\frac{1}{L_{i}} .
\end{aligned}
$$

From here on, we let $\bar{\rho}=\max _{i \in \mathcal{I}_{\mathrm{B}}} \bar{\rho}_{i}$ and $\bar{L}=\max _{i \in \mathcal{I}_{\mathrm{F}}} L_{i}$ with the convention that $\bar{\rho}=0$ if $\mathcal{I}_{\mathrm{B}}=\{\emptyset\}$ and $\bar{L}=0$ if $\mathcal{I}_{\mathrm{F}}=\{\emptyset\}$.

The recent work [15] includes several extensions to the basic forward-step stepsize constraint (16), under which one still obtains weak convergence of the iterates to a solution. Section 4.1 of [15] presents a backtracking linesearch that may be used when the constant $L_{i}$ is unknown. Section 4.2 of [15] presents a backtrack-free adaptive stepsize for the special case in which $T_{i}$ is affine but $L_{i}$ is unknown. Our convergence rate analysis holds for these extensions, but for the sake of readability we omit the details.

\section{Results Similar to [15]}

Lemma 4. Suppose Assumption [1 holds. In the context of Algorithm 1, recall the notation $p^{k}=\left(z^{k}, w_{1}^{k}, \ldots, w_{n-1}^{k}\right)^{\top}$. For all $1 \leq k<\bar{K}$ :

1. The iterates can be written as

$$
p^{k+1}=p^{k}-\alpha_{k} \nabla \varphi_{k}\left(p^{k}\right)
$$

where $\alpha_{k}=\beta_{k} \varphi_{k}\left(p^{k}\right) /\left\|\nabla \varphi_{k}\right\|$ and $\varphi_{k}$ is defined in (8)).

2. For all $p^{*}=\left(z^{*}, w_{1}^{*}, \ldots, w_{n-1}^{*}\right) \in \mathcal{S}$ :

$$
\left\|p^{k+1}-p^{*}\right\|^{2} \leq\left\|p^{k}-p^{*}\right\|^{2}-\beta_{k}\left(2-\beta_{k}\right)\left\|p^{k+1}-p^{k}\right\|^{2}
$$

which implies that

$$
\begin{aligned}
& \sum_{t=1}^{k}\left\|p^{t}-p^{t+1}\right\|^{2} \leq \tau\left\|p^{1}-p^{*}\right\|^{2}, \quad \text { where } \quad \tau=\underline{\beta}^{-1}(2-\bar{\beta})^{-1} \\
& \left\|z^{k}-z^{*}\right\| \leq \gamma^{-\frac{1}{2}}\left\|p^{k}-p^{*}\right\| \leq \gamma^{-\frac{1}{2}}\left\|p^{1}-p^{*}\right\|, \\
& \left\|w_{i}^{k}-w_{i}^{*}\right\| \leq\left\|p^{k}-p^{*}\right\| \leq\left\|p^{1}-p^{*}\right\|, \quad i=1, \ldots, n-1 \text {. }
\end{aligned}
$$

Proof. The update (17) follows from algebraic manipulation of lines 1020 and consideration of (8) and (9). Inequalities (18) and (19) result from Algorithm 1 being a separator-projector algorithm [15, Lemma 6]. A specific reference proving these results is [10, Proposition 1]. 
The following lemma places an upper bound on the gradient of the affine function $\varphi_{k}$ at each iteration. A similar result was proved in [15, Lemma 11], but since that result is slightly different, we include the full proof here.

Lemma 5. Suppose assumptions 10,2, and 3 hold and recall the affine function $\varphi_{k}$ defined in (8). For all $1 \leq k \leq \bar{K}$,

$$
\left\|\nabla \varphi_{k}\right\|^{2} \leq \xi_{1} \sum_{i=1}^{n}\left\|z^{k}-x_{i}^{k}\right\|^{2}
$$

where

$$
\xi_{1}=2 n\left[1+2 \gamma^{-1}\left(\bar{L}^{2}\left|\mathcal{I}_{\mathrm{F}}\right|+\underline{\rho}^{-2}(1+\delta)\right)\right]<\infty .
$$

Proof. Using Lemma 3 ,

$$
\left\|\nabla \varphi_{k}\right\|^{2}=\gamma^{-1}\left\|\sum_{i=1}^{n} y_{i}^{k}\right\|^{2}+\sum_{i=1}^{n-1}\left\|x_{i}^{k}-x_{n}^{k}\right\|^{2} .
$$

Using Lemma 1, we begin by writing the second term on the right of (23) as

$$
\sum_{i=1}^{n-1}\left\|x_{i}^{k}-x_{n}^{k}\right\|^{2} \leq 2 \sum_{i=1}^{n-1}\left(\left\|x_{i}^{k}-z^{k}\right\|^{2}+\left\|z^{k}-x_{n}^{k}\right\|^{2}\right) \leq 2 n \sum_{i=1}^{n}\left\|z^{k}-x_{i}^{k}\right\|^{2} .
$$

We next consider the first term in (23). Rearranging the update equations for Algorithm 1 as given in lines 5 and 8 , we may write

$$
\begin{aligned}
y_{i}^{k} & =\left(\rho_{i}^{k}\right)^{-1}\left(z^{k}-x_{i}^{k}+\rho_{i}^{k} w_{i}^{k}+e_{i}^{k}\right), & & i \in \mathcal{I}_{\mathrm{B}} \\
T_{i} z^{k} & =\left(\rho_{i}^{k}\right)^{-1}\left(z^{k}-x_{i}^{k}+\rho_{i}^{k} w_{i}^{k}\right), & & i \in \mathcal{I}_{\mathrm{F}} .
\end{aligned}
$$

Note that (25) rewrites line 5 of Algorithm 1 using (3). The first term of (23) may then be written as

$$
\begin{aligned}
&\left\|\sum_{i=1}^{n} y_{i}^{k}\right\|^{2}=\left\|\sum_{i \in \mathcal{I}_{\mathrm{B}}} y_{i}^{k}+\sum_{i \in \mathcal{I}_{\mathrm{F}}}\left(T_{i} z^{k}+y_{i}^{k}-T_{i} z^{k}\right)\right\|^{2} \\
& \stackrel{(\text { a) }}{\leq} 2\left\|\sum_{i \in \mathcal{I}_{\mathrm{B}}} y_{i}^{k}+\sum_{i \in \mathcal{I}_{\mathrm{F}}} T_{i} z^{k}\right\|^{2}+2\left\|\sum_{i \in \mathcal{I}_{\mathrm{F}}}\left(y_{i}^{k}-T_{i} z^{k}\right)\right\|^{2} \\
& \stackrel{(\text { b) }}{=} 2\left\|\sum_{i=1}^{n}\left(\rho_{i}^{k}\right)^{-1}\left(z^{k}-x_{i}^{k}+\rho_{i}^{k} w_{i}^{k}\right)+\sum_{i \in \mathcal{I}_{\mathrm{B}}}\left(\rho_{i}^{k}\right)^{-1} e_{i}^{k}\right\|^{2} \\
& \quad+2\left\|\sum_{i \in \mathcal{I}_{\mathrm{F}}}\left(T x_{i}^{k}-T_{i} z^{k}\right)\right\|^{2} \\
& \stackrel{\text { (c) }}{\leq} 4\left\|\sum_{i=1}^{n}\left(\rho_{i}^{k}\right)^{-1}\left(z^{k}-x_{i}^{k}+\rho_{i}^{k} w_{i}^{k}\right)\right\|^{2}+4\left\|\sum_{i \in \mathcal{I}_{\mathrm{B}}}\left(\rho_{i}^{k}\right)^{-1} e_{i}^{k}\right\|^{2}
\end{aligned}
$$




$$
\begin{aligned}
& +2\left|\mathcal{I}_{\mathrm{F}}\right| \sum_{i \in \mathcal{I}_{\mathrm{F}}}\left\|T_{i} x_{i}^{k}-T_{i} z^{k}\right\|^{2} \\
& \stackrel{(\mathrm{d})}{\leq} 4 n \underline{\rho}^{-2}\left(\sum_{i=1}^{n}\left\|z^{k}-x_{i}^{k}\right\|^{2}+\sum_{i \in \mathcal{I}_{\mathrm{B}}}\left\|e_{i}^{k}\right\|^{2}\right)+2\left|\mathcal{I}_{\mathrm{F}}\right| \sum_{i \in \mathcal{I}_{\mathrm{F}}}\left(L_{i}^{2}\left\|x_{i}^{k}-z^{k}\right\|^{2}\right) \\
& \stackrel{\text { (e) }}{\leq} \xi_{1}^{\prime} \sum_{i=1}^{n}\left\|z^{k}-x_{i}^{k}\right\|^{2}
\end{aligned}
$$

where

$$
\xi_{1}^{\prime}=4 n\left(\bar{L}^{2}\left|\mathcal{I}_{\mathrm{F}}\right|+\underline{\rho}^{-2}(1+\delta)\right)
$$

where recall $\bar{L}=\max _{i \in \mathcal{I}_{\mathrm{F}}} L_{i}$. In the above, (a) uses Lemma 1, while (b) is obtained by substituting (25)-(26) into the first squared norm and using $y_{i}^{k}=T_{i} x_{i}^{k}$ for $i \in \mathcal{I}_{\mathrm{F}}$ in the second. Next, (c) uses Lemma 1 1 once more on both terms. Inequality (d) uses Lemma 1, the Lipschitz continuity of $T_{i}, \sum_{i=1}^{n} w_{i}^{k}=0$, and Assumption 3. Finally, (e) follows by collecting terms and using Assumption 2. Combining (23), (24), and (27) establishes the Lemma with $\xi_{1}$ as defined in (22).

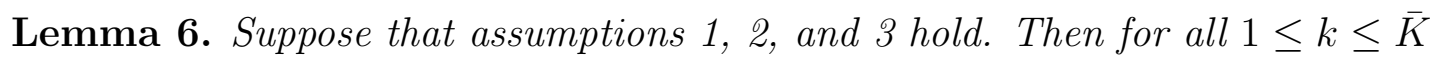

$$
\varphi_{k}\left(p^{k}\right) \geq \xi_{2} \sum_{i=1}^{n}\left\|z^{k}-x_{i}^{k}\right\|^{2} .
$$

where

$$
\xi_{2}=\min \left\{(1-\sigma) \bar{\rho}^{-1}, \min _{j \in \mathcal{I}_{\mathrm{F}}}\left\{\bar{\rho}_{j}^{-1}-L_{j}\right\}\right\}>0 .
$$

Furthermore, for all such $k$,

$$
\varphi_{k}\left(p^{k}\right)+\sum_{i \in \mathcal{I}_{\mathrm{F}}} L_{i}\left\|z^{k}-x_{i}^{k}\right\|^{2} \geq(1-\sigma) \underline{\rho} \sum_{i \in \mathcal{I}_{\mathrm{B}}}\left\|y_{i}^{k}-w_{i}^{k}\right\|^{2}+\underline{\rho} \sum_{i \in \mathcal{I}_{\mathrm{F}}}\left\|T_{i} z^{k}-w_{i}^{k}\right\|^{2} .
$$

Proof. The claimed results are special cases of those given in lemmas 12-13 of [15].

\section{New Lemmas Needed to Derive Convergence Rates}

Lemma 7. Suppose assumptions 1, 2, and 3 hold, and recall $\alpha_{k}$ computed on line 15 of Algorithm 1. For all $1 \leq k<\bar{K}$, it holds that $\alpha_{k} \geq \underline{\alpha} \triangleq \beta_{2} / \xi_{1}>0$, where $\xi_{1}$ and $\xi_{2}$ are as defined in (22) and (28).

Proof. By Lemma 4, $\alpha_{k}$ defined on line 15 of Algorithm 1 may be expressed as

$$
\alpha_{k}=\frac{\beta_{k} \varphi_{k}\left(z^{k}, \mathbf{w}^{k}\right)}{\left\|\nabla \varphi_{k}\right\|^{2}} .
$$


By Lemma 5, $\left\|\nabla \varphi_{k}\right\|^{2} \leq \xi_{1} \sum_{i=1}^{n}\left\|z^{k}-x_{i}^{k}\right\|^{2}$, where $\xi_{1}$ is defined in (22). Furthermore, Lemma 6 implies that $\varphi_{k}\left(z^{k}, \mathbf{w}^{k}\right) \geq \xi_{2} \sum_{i=1}^{n}\left\|z^{k}-x_{i}^{k}\right\|^{2}$, where $\xi_{2}$ is defined in (28). Combining these two inequalities with (30) and $\beta_{k} \geq \beta$ yields $\alpha_{k} \geq \beta \xi_{1} / \xi_{2}=\underline{\alpha}$. Using (22) and (28), $\xi_{1}$ and $\xi_{2}$ are positive and finite by assumptions 2 and 3 . Since $\beta_{k}>\underline{\beta}>0$, we conclude that $\underline{\alpha}>0$.

The next lemma is the key to proving $\mathrm{O}(1 / k)$ function value convergence rate and linear convergence rate under strong mononotonicity and cocoercivity. Essentially, it shows that several key quanitities of Algorithm 1 are square-summable, within known bounds.

Lemma 8. Suppose assumptions 1, 2, and 3 hold. If $\bar{K}=\infty$, then $\varphi_{k}\left(p^{k}\right) \rightarrow 0$ and $\nabla \varphi_{k} \rightarrow 0$. Furthermore, for all $1 \leq k<\bar{K}$,

$$
\begin{gathered}
\sum_{t=1}^{k}\left\|z^{t+1}-z^{t}\right\|^{2} \leq \gamma^{-1} \tau\left\|p^{1}-p^{*}\right\|^{2}, \\
\sum_{t=1}^{k} \sum_{i=1}^{n-1}\left\|w_{i}^{t+1}-w_{i}^{t}\right\|^{2} \leq \tau\left\|p^{1}-p^{*}\right\|^{2}, \\
\sum_{i=1}^{n}\left\|z^{k}-x_{i}^{k}\right\|^{2} \leq \frac{\xi_{1}}{\underline{\beta}^{2} \xi_{2}^{2}}\left\|p^{k+1}-p^{k}\right\|^{2}, \quad \sum_{t=1}^{k} \sum_{i=1}^{n}\left\|z^{t}-x_{i}^{t}\right\|^{2} \leq \frac{\tau \xi_{1}}{\underline{\beta}^{2} \xi_{2}^{2}}\left\|p^{1}-p^{*}\right\|^{2}, \\
\sum_{i=1}^{n}\left\|w_{i}^{k}-y_{i}^{k}\right\|^{2} \leq E_{1}\left\|p^{k+1}-p^{k}\right\|^{2}, \quad \sum_{t=1}^{n} \sum_{i=1}^{n}\left\|w_{i}^{t}-y_{i}^{t}\right\|^{2} \leq \tau E_{1}\left\|p^{1}-p^{*}\right\|^{2}, \\
\sum_{t=1}^{k} \sum_{i \in \mathcal{I}_{\mathrm{F}}}\left\|w_{i}^{t}-T_{i} z^{t}\right\|^{2} \leq \tau E_{1}\left\|p^{1}-p^{*}\right\|^{2},
\end{gathered}
$$

where

$$
E_{1}=2(1-\sigma)^{-1} \underline{\rho}^{-1}\left(1+\xi_{2}^{-1} \bar{L}(1+\underline{\rho} \bar{L})\right) \frac{\xi_{1}}{\underline{\beta}^{2} \xi_{2}},
$$

$\tau$ is as defined in (19), and $\xi_{1}$ and $\xi_{2}$ are as defined in (22) and (28).

Proof. Fix any $1 \leq k<\bar{K}$. First, from (19) in Lemma 4, we have $\sum_{t=1}^{k}\left\|p^{t+1}-p^{t}\right\|^{2} \leq$ $\tau\left\|p^{1}-p^{*}\right\|^{2}$. Since $\left\|p^{k+1}-p^{k}\right\|^{2}=\gamma\left\|z^{k+1}-z^{k}\right\|^{2}+\sum_{i=1}^{n-1}\left\|w_{i}^{k+1}-w_{i}^{k}\right\|^{2}$, inequalities (31) and (32) follow immediately. Next, Lemma 4 also implies that $p^{k+1}-p^{k}=-\left(\beta_{k} \varphi_{k}\left(p^{k}\right) /\left\|\nabla \varphi_{k}\right\|^{2}\right) \nabla \varphi_{k}$, and therefore that

$$
\left\|p^{k+1}-p^{k}\right\|=\frac{\beta_{k} \varphi_{k}\left(p^{k}\right)}{\left\|\nabla \varphi_{k}\right\|} .
$$

As argued in Lemma 7, lemmas 5 and 6 imply that

$$
\frac{\varphi_{k}\left(p^{k}\right)}{\left\|\nabla \varphi_{k}\right\|^{2}} \geq \frac{\xi_{2}}{\xi_{1}} \quad \Longrightarrow \quad\left\|\nabla \varphi_{k}\right\|^{2} \leq \frac{\xi_{1}}{\xi_{2}} \varphi_{k}\left(p^{k}\right) .
$$


Since $\varphi_{k}\left(p^{k}\right) \geq 0$ by Lemma 3, substituting (38) into (37) and using the lower bound on $\beta_{k}$ yields

$$
\varphi_{k}\left(p^{k}\right)=\beta_{k}^{-1}\left\|\nabla \varphi_{k}\right\|\left\|p^{k+1}-p^{k}\right\| \leq \underline{\beta}^{-1}\left(\frac{\xi_{1}}{\xi_{2}}\right)^{\frac{1}{2}} \sqrt{\varphi_{k}\left(p^{k}\right)}\left\|p^{k+1}-p^{k}\right\|,
$$

which in turn leads to

$$
\sqrt{\varphi_{k}\left(p^{k}\right)} \leq \underline{\beta}^{-1}\left(\frac{\xi_{1}}{\xi_{2}}\right)^{\frac{1}{2}}\left\|p^{k+1}-p^{k}\right\| \quad \Longrightarrow \quad \varphi_{k}\left(p^{k}\right) \leq \frac{\xi_{1}}{\xi_{2} \underline{\beta}^{2}}\left\|p^{k+1}-p^{k}\right\|^{2} .
$$

Therefore,

$$
\sum_{t=1}^{k} \varphi_{t}\left(p^{t}\right) \leq \frac{\xi_{1}}{\xi_{2} \underline{\beta}^{2}} \sum_{t=1}^{k}\left\|p^{t+1}-p^{t}\right\|^{2} \leq \frac{\tau \xi_{1}}{\underline{\beta}^{2} \xi_{2}}\left\|p^{1}-p^{*}\right\|^{2} .
$$

If $\bar{K}=\infty$, (40) implies that $\varphi_{k}\left(p^{k}\right) \rightarrow 0$, which in conjunction with (38) implies that $\nabla \varphi_{k} \rightarrow 0$. Combining (39) with Lemma 6 yields the first part of (33). Applying (19) from Lemma 4 then yields the second part of (33).

Finally, we establish (34) and (35). Inequality (29) from Lemma 6 implies that

$$
\begin{aligned}
(1-\sigma) \underline{\rho} \sum_{i \in \mathcal{I}_{\mathrm{B}}}\left\|w_{i}^{k}-y_{i}^{k}\right\|^{2}+\underline{\rho} \sum_{i \in \mathcal{I}_{\mathrm{F}}}\left\|w_{i}^{k}-T_{i} z^{k}\right\|^{2} & \leq \varphi_{k}\left(p^{k}\right)+\bar{L} \sum_{i \in \mathcal{I}_{\mathrm{F}}}\left\|x_{i}^{k}-z^{k}\right\|^{2} \\
& \leq\left(1+\xi_{2}^{-1} \bar{L}\right) \frac{\xi_{1}}{\underline{\beta}^{2} \xi_{2}}\left\|p^{k+1}-p^{k}\right\|^{2}
\end{aligned}
$$

where in the second inequality we have used (39) and (33). Together with (19) from Lemma4, (41) implies (35) . Furthermore, for any $i \in \mathcal{I}_{\mathrm{F}}$, we may write $w_{i}^{k}-y_{i}^{k}=w_{i}^{k}-T_{i} z^{k}+T_{i} z^{k}-y_{i}^{k}$, from which Lemma 1 can be used to obtain

$$
\left\|w_{i}^{k}-T_{i} z^{k}\right\|^{2} \geq \frac{1}{2}\left\|w_{i}^{k}-y_{i}^{k}\right\|^{2}-\left\|T_{i} z^{k}-y_{i}^{k}\right\|^{2} .
$$

Substituting (42) into the left hand side of (41) yields

$$
\begin{aligned}
& (1-\sigma) \underline{\rho} \sum_{i \in \mathcal{I}_{\mathrm{B}}}\left\|w_{i}^{k}-y_{i}^{k}\right\|^{2}+\frac{1}{2} \underline{\rho} \sum_{i \in \mathcal{I}_{\mathrm{F}}}\left\|w_{i}^{k}-y_{i}^{k}\right\|^{2} \\
& \leq\left(1+\xi_{2}^{-1} \bar{L}\right) \frac{\xi_{1}}{\underline{\beta}^{2} \xi_{2}}\left\|p^{k+1}-p^{k}\right\|^{2}+\underline{\rho} \sum_{i \in \mathcal{I}_{\mathrm{F}}}\left\|T_{i} z^{k}-T_{i} x_{i}^{k}\right\|^{2} \\
& \leq\left(1+\xi_{2}^{-1} \bar{L}\right) \frac{\xi_{1}}{\underline{\beta}^{2} \xi_{2}}\left\|p^{k+1}-p^{k}\right\|^{2}+\underline{\rho} \bar{L}^{2} \sum_{i \in \mathcal{I}_{\mathrm{F}}}\left\|z^{k}-x_{i}^{k}\right\|^{2} \\
& \leq\left(1+\xi_{2}^{-1} \bar{L}(1+\underline{\rho} \bar{L})\right) \frac{\xi_{1}}{\underline{\beta}^{2} \xi_{2}}\left\|p^{k+1}-p^{k}\right\|^{2}
\end{aligned}
$$

where first inequality follows by substituting $y_{k}^{k}=T_{i} x_{i}^{k}$ for $i \in \mathcal{I}_{\mathrm{F}}$, the second inequality uses the Lipschitz continuity of $T_{i}$ for $i \in \mathcal{I}_{\mathrm{F}}$, and the final inequality uses (33). Because 
$0 \leq \sigma<1$, we may replace the coefficients in front of the two left-hand sums by the $(1-\sigma) / 2$, which can only be smaller, and obtain

$$
\sum_{i=1}^{n}\left\|w_{i}^{k}-y_{i}^{k}\right\|^{2} \leq 2(1-\sigma)^{-1} \underline{\rho}^{-1}\left(1+\xi_{2}^{-1} \bar{L}(1+\underline{\rho} \bar{L})\right) \frac{\xi_{1}}{\xi_{2}}\left\|p^{k+1}-p^{k}\right\|^{2}
$$

which yields the first part of (34). The second part follows by applying (19) to (43).

The final technical lemma shows that the sequences $\left\{x_{i}^{k}\right\}$ and $\left\{y_{i}^{k}\right\}$ are bounded for $i=$ $1, \ldots, n$, and computes specific bounds on their norms.

Lemma 9. Suppose assumptions 1, 2, and [3 hold. The sequences $\left\{x_{i}^{k}\right\}$ and $\left\{y_{i}^{k}\right\}$ are bounded for $i=1, \ldots, n$. In particular, for all $i=1, \ldots, n$, and $1 \leq k \leq \bar{K}$,

$$
\begin{array}{ll}
\left\|x_{i}^{k}\right\|^{2} \leq \min _{p^{*} \in \mathcal{S}}\left\{2\left(\frac{\xi_{1}}{\xi_{2}^{2}}+\gamma^{-1}\right)\left\|p^{1}-p^{*}\right\|^{2}+2 \gamma^{-1}\left\|p^{*}\right\|^{2}\right\} & \triangleq B_{x}^{2} \\
\left\|y_{i}^{k}\right\|^{2} \leq \min _{p^{*} \in \mathcal{S}}\left\{2\left(n+E_{1}\right)\left\|p^{1}-p^{*}\right\|^{2}+2 n\left\|p^{*}\right\|^{2}\right\} & \triangleq B_{y}^{2} .
\end{array}
$$

Proof. Assumption 1 asserts $\mathcal{S}$ is nonempty, so let $p^{*} \in \mathcal{S}$ and fix any $1 \leq k<\bar{K}$. From Lemma 1 .

$$
\left\|p^{k}\right\|^{2}=\left\|p^{k}-p^{*}+p^{*}\right\|^{2} \leq 2\left\|p^{k}-p^{*}\right\|^{2}+2\left\|p^{*}\right\|^{2} \leq 2\left\|p^{1}-p^{*}\right\|^{2}+2\left\|p^{*}\right\|^{2},
$$

where the final inequality uses (18). It immediately follows that

$$
\begin{aligned}
\left\|z^{k}\right\|^{2} & \leq \gamma^{-1}\left\|p^{k}\right\|^{2} \leq 2 \gamma^{-1}\left\|p^{1}-p^{*}\right\|^{2}+2 \gamma^{-1}\left\|p^{*}\right\|^{2} \\
\sum_{i=1}^{n-1}\left\|w_{i}^{k}\right\|^{2} & \leq\left\|p^{k}\right\|^{2} \leq 2\left\|p^{1}-p^{*}\right\|^{2}+2\left\|p^{*}\right\|^{2}
\end{aligned}
$$

Furthermore, using the definition of $w_{n}^{k}$ and Lemma 1, we also have

$$
\left\|w_{n}^{k}\right\|^{2}=\left\|\sum_{i=1}^{n-1} w_{i}^{k}\right\|^{2} \leq(n-1) \sum_{i=1}^{n-1}\left\|w_{i}^{k}\right\|^{2} \leq 2 n\left\|p^{1}-p^{*}\right\|^{2}+2 n\left\|p^{*}\right\|^{2} .
$$

Therefore, for all $i=1, \ldots, n$, we have

$$
\left\|x_{i}^{k}\right\|^{2} \leq 2\left\|z^{k}-x_{i}^{k}\right\|^{2}+2\left\|z^{k}\right\|^{2} \leq 2\left(\frac{\xi_{1}}{\underline{\beta}^{2} \xi_{2}^{2}}+\gamma^{-1}\right)\left\|p^{1}-p^{*}\right\|^{2}+2 \gamma^{-1}\left\|p^{*}\right\|^{2},
$$

where second inequality uses (47) and (33). Finally, for $i=1, \ldots, n$

$$
\left\|y_{i}^{k}\right\|^{2} \leq 2\left\|y_{i}^{k}-w_{i}^{k}\right\|^{2}+2\left\|w_{i}^{k}\right\|^{2} \leq 2\left(n+E_{1}\right)\left\|p^{1}-p^{*}\right\|^{2}+2 n\left\|p^{*}\right\|^{2},
$$

where the second inequality uses (34) and (48)-(49). Note that the factor of $n$ is only necessary when $i=n$, but for simplicity we will just a single bound for all $i$.

Finally, the set $\mathcal{S}$ is closed and convex from Proposition 1, and the expressions (50) and (51) are continuous functions of $p^{*}$. Thus, we may minimize these bounds over $\mathcal{S}$, yielding (44)-(45) . 


\section{Function Value Convergence Rate}

\subsection{Assumptions}

We now consider the optimization problem given in (2), which we repeat here:

$$
F^{*}=\min _{z \in \mathcal{H}} F(z)=\min _{z \in \mathcal{H}} \sum_{i=1}^{n} f_{i}(z) .
$$

Assumption 4. Problem (52) conforms to the following:

1. $\mathcal{H}$ is a real Hilbert space.

2. Each $f_{i}: \mathcal{H} \rightarrow(-\infty,+\infty]$ is convex, closed, and proper.

3. There exists some subset $\mathcal{I}_{\mathrm{F}} \subseteq\{1, \ldots, n\}$ s.t. for all $i \in \mathcal{I}_{\mathrm{F}}, f$ is Fréchet differentiable everywhere and $\nabla f_{i}$ is $L_{i}$-Lipschitz continuous.

4. For $i \in \mathcal{I}_{\mathrm{B}} \triangleq\{1, \ldots, n\} \backslash \mathcal{I}_{\mathrm{F}}$, $\operatorname{prox}_{\rho f_{i}}$ can be computed to within the error tolerance specified in Assumption 2 (however, these functions are not precluded from also having Lipschitz continuous gradients).

5. Letting $T_{i}=\partial f_{i}$ for $i=1, \ldots, n$, the solution set $\mathcal{S}$ in (6) is nonempty.

When $\left|\mathcal{I}_{\mathrm{B}}\right| \geq 1$, it is possible for (52) to have a finite solution, yet for $\mathcal{S}$ to be empty. For constraint-qualification-like conditions precluding such pathological situations, see for example [2, Corollary 16.50].

Lemma 10. If Assumption 4 holds, then Assumption 1 holds for (1) with $T_{i}=\partial f_{i}$ for $i=1, \ldots, n$. If $(z, \mathbf{w}) \in \mathcal{S}$, then $z$ is a solution to (52). The solution value $F^{*}$ is finite.

Proof. Closed, convex, and proper functions have maximal monotone subdifferentials [2, Theorem 21.2]. That $z$ is a solution to (52) for any $(z, \mathbf{w}) \in \mathcal{S}$ is a consequence of Fermat's rule [2, Proposition 27.1] and the elementary fact that $\sum_{i} \partial f_{i}(x) \subseteq \partial\left(\sum_{i} f_{i}\right)(x)$. Since $\mathcal{S}$ is nonempty, a solution to (52) exists. For any $(z, \mathbf{w}) \in \mathcal{S}$, the function $f_{i}$ must be subdifferentiable and hence finite at $z$ for all $i=1, \ldots, n$, so $F^{*}=\sum_{i=1}^{n} f_{i}(z)$ must be finite.

In the following analysis, we consider two types of convergence rates. First we will establish a rate of the form

$$
\sum_{i=1}^{n} f_{i}\left(\bar{x}_{i}^{k}\right)-F^{*}=\mathrm{O}(1 / k) \quad \text { and } \quad\left\|\bar{x}_{i}^{k}-\bar{x}_{j}^{k}\right\|=\mathrm{O}(1 / k) \quad \forall i, j=1, \ldots, n,
$$

where $\bar{x}_{i}^{k}$ is an appropriate averaging of the sequence $\left\{x_{i}^{k}\right\}$. With an additional Lipschitz continuity assumption, we can derive a more direct rate of the form

$$
\sum_{i=1}^{n} f_{i}\left(\bar{x}_{j}^{k}\right)-F^{*}=\mathrm{O}(1 / k)
$$

For an appropriate index $j$. This additional assumption is as follows: 
Assumption 5. If $\left|\mathcal{I}_{\mathrm{B}}\right|>1$, there exists $\mathcal{I}_{\mathrm{L}} \subseteq \mathcal{I}_{\mathrm{B}}$ such that $\left|\mathcal{I}_{\mathrm{L}}\right| \geq\left|\mathcal{I}_{\mathrm{B}}\right|-1$, and for all $i \in \mathcal{I}_{\mathrm{L}}$ the function $f_{i}$ is $M_{i}$-Lipschitz continuous on the ball: $\left\{x:\|x\| \leq B_{x}\right\}$ where $B_{x}$ is defined in (44).

By virtue of Assumption [5, note that for all $i \in \mathcal{I}_{\mathrm{L}},\left\{x:\|x\| \leq B_{x}\right\} \subseteq \operatorname{dom}\left(f_{i}\right)$.

We point out that it is not surprising that Assumption 5 is required to derive convergence rates of the form (53): suppose the first two functions $f_{1}$ and $f_{2}$ are the respective indicator functions of closed nonempty convex sets $\mathcal{C}_{1}$ and $\mathcal{C}_{2}$, that is, $f_{i}(x)=0$ if $x \in \mathcal{C}_{i}$ and otherwise $f_{i}(x)=+\infty$. Since neither function is differentiable, $\{1,2\} \subseteq \mathcal{I}_{\mathrm{B}}$. Since neither function is Lipschitz continuous, $\{1,2\} \notin \mathcal{I}_{\mathrm{L}}$, unless $B_{x} \subseteq \mathcal{C}_{1} \cap \mathcal{C}_{2}$. This last situation is of little interest, since the iterates remain inside $\mathcal{C}_{1} \cap \mathcal{C}_{2}$ for all iterations and thus the constraints encoded by $f_{1}$ and $f_{2}$ may be disregarded.

Instead, suppose $B_{x} \not \mathcal{C}_{1} \cap \mathcal{C}_{2}$ and thus $\{1,2\} \notin \mathcal{I}_{\mathrm{L}}$. Then $\left|\mathcal{I}_{\mathrm{L}}\right| \leq\left|\mathcal{I}_{\mathrm{B}}\right|-2$, which violates Assumption 5. Suppose anyway that we could establish a convergence rate of $\mathrm{O}(1 / k)$ (or similar) for some sequence of points $\tilde{x}^{k}$ generated by the algorithm. But this would imply that $\tilde{x}^{k} \in \mathcal{C}_{1} \cap \mathcal{C}_{2}$ for all $k$, meaning that we would have to be able to solve an arbitrary two-set convex feasibility problem in a single iteration. Of course, it is easy to construct a counterexample in which Algorithm 1 does not find a point in the intersection of two closed convex sets within a finite number of iterations.

So, to derive a convergence rate of the form (53) we allow for only one function to be non-Lipschitz and thus able to encode a "hard" constraint. Any other nonsmooth functions present in the problem must be Lipschitz continuous on the bounded set $B_{x}$ which contains all points potentially encountered by the algorithm.

\subsection{Main Result}

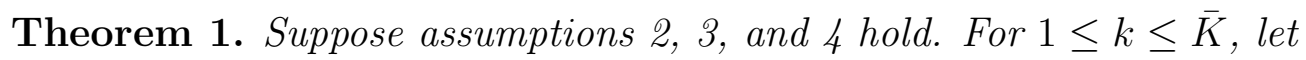

$$
(\forall i=1, \ldots, n) \quad \bar{x}_{i}^{k}=\frac{\sum_{t=1}^{k} \alpha_{t} x_{i}^{t}}{\sum_{t=1}^{k} \alpha_{t}},
$$

where $\alpha_{t}$ is calculated on line 15 of Algorithm 1 . Fixing any $p^{*} \in \mathcal{S}$, one has

1. If $\bar{K}<\infty$,

$$
(\forall j=1, \ldots, n) \quad \sum_{i=1}^{n} f_{i}\left(x_{j}^{\bar{K}}\right)=\sum_{i=1}^{n} f_{i}\left(z^{\bar{K}+1}\right)=F^{*},
$$

2. For all $1 \leq k<\bar{K}$

$$
\sum_{i=1}^{n} f_{i}\left(\bar{x}_{i}^{k}\right)-F^{*} \leq \frac{E_{2}\left\|p^{1}-p^{*}\right\|^{2}+E_{3}\left\|p^{1}-p^{*}\right\|}{k}
$$

where

$$
E_{2}=\frac{\xi_{1}}{2 \underline{\beta} \xi_{2}}\left(1+\left(3+2 E_{1}\right) \tau+\bar{\rho}_{n} \tau\left(2+\gamma E_{1}+\frac{\gamma \delta \xi_{1}}{\underline{\beta}^{2} \xi_{2}^{2}}\right)\right)
$$




$$
E_{3}=2 \sqrt{n}\left\|p^{*}\right\| \frac{\xi_{1}}{\beta \xi_{2}}
$$

Furthermore,

$$
(\forall i, l=1, \ldots, n) \quad\left\|\bar{x}_{i}^{k}-\bar{x}_{l}^{k}\right\| \leq \frac{4\left\|p^{1}-p^{*}\right\|}{\underline{\alpha} k}=\frac{4 \xi_{1}\left\|p^{1}-p^{*}\right\|}{\underline{\beta} \xi_{2} k} .
$$

3. Additionally suppose Assumption 5 holds. If $\mathcal{I}_{\mathrm{B}}=1$, let $j$ be the unique element in $\mathcal{I}_{\mathrm{B}}$. If $\left|\mathcal{I}_{\mathrm{B}}\right|>1$, let $j$ be the unique element of $\mathcal{I}_{\mathrm{B}} \backslash \mathcal{I}_{\mathrm{L}}$. If $\left|\mathcal{I}_{\mathrm{B}}\right|=0$ then choose $j$ to be any index in $\mathcal{I}_{\mathrm{F}}$. Then, for all $1 \leq k<\bar{K}$,

$$
\sum_{i=1}^{n} f_{i}\left(\bar{x}_{j}^{k}\right)-F^{*} \leq \frac{E_{2}\left\|p^{1}-p^{*}\right\|^{2}+E_{4}\left\|p^{1}-p^{*}\right\|}{k}
$$

where

$$
E_{4}=E_{3}+\frac{4 \xi_{1}}{\underline{\beta} \xi_{2}}\left(\sum_{i \in \mathcal{I}_{\mathrm{L}}} M_{i}+n B_{y}\left(1+2 \bar{L} B_{x}\right)\right)
$$

Proof. In the case that $\bar{K}<\infty$, it was established in [15, Lemma 5] that $x_{1}^{\bar{K}}=\cdots=x_{n}^{\bar{K}}=$ $z^{\bar{K}+1}$ is a solution to (52), which establishes (54). We now address points 2 and 3 .

Part 1: An upper bound for $\sum_{i=1}^{n} f_{i}\left(\bar{x}_{i}^{k}\right)-F^{*}$

We begin by establishing the upper bound on $\sum_{i=1}^{n} f_{i}\left(\bar{x}_{i}^{k}\right)-F^{*}$ in (55). Since $f_{i}$ is convex,

$$
\sum_{i=1}^{n} f_{i}\left(\bar{x}_{i}^{k}\right)-F^{*}=\sum_{i=1}^{n} f_{i}\left(\frac{\sum_{t=1}^{k} \alpha_{t} x_{i}^{t}}{\sum_{t=1}^{k} \alpha_{t}}\right)-F^{*} \leq \frac{\sum_{t=1}^{k} \alpha_{t}\left(\sum_{i=1}^{n} f_{i}\left(x_{i}^{t}\right)-F^{*}\right)}{\sum_{t=1}^{k} \alpha_{t}}
$$

Since Lemma 7 implies that $\alpha_{k} \geq \underline{\alpha}$, we will aim to show that $\sum_{t=1}^{k} \alpha_{t}\left(\sum_{i=1}^{n} f_{i}\left(x_{i}^{t}\right)-F^{*}\right)$ is bounded for all $1 \leq k<\bar{K}$ (recall that $\bar{K}$ may be $+\infty$ ).

The projection updates on lines [18, 19 of Algorithm 1 mean that, for all $1 \leq k<\bar{K}$,

$$
\begin{aligned}
& z^{k+1}=z^{k}-\gamma^{-1} \alpha_{k} \sum_{i=1}^{n} y_{i}^{k} \\
& w_{i}^{k+1}=w_{i}^{k}-\alpha_{k}\left(x_{i}^{k}-x_{n}^{k}\right), \quad i=1, \ldots, n-1 .
\end{aligned}
$$

Take any $p^{*}=(x, \mathbf{w}) \in \mathcal{S}$. By Lemma $10, F^{*}=\sum_{i=1}^{n} f_{i}(x)$. Then

$$
\begin{aligned}
\sum_{i=1}^{n} f_{i}\left(x_{i}^{k}\right)-F^{*} & =\sum_{i=1}^{n} f_{i}\left(x_{i}^{k}\right)-\sum_{i=1}^{n} f_{i}(x) \\
& \stackrel{(a)}{\leq} \sum_{i=1}^{n}\left\langle y_{i}^{k}, x_{i}^{k}-x\right\rangle
\end{aligned}
$$




$$
\begin{aligned}
& =\left\langle\sum_{i=1}^{n} y_{i}^{k}, x_{n}^{k}-x\right\rangle+\sum_{i=1}^{n-1}\left\langle y_{i}^{k}, x_{i}^{k}-x_{n}^{k}\right\rangle \\
& \stackrel{(b)}{=} \underbrace{\frac{\gamma}{\alpha_{k}}\left\langle z^{k}-z^{k+1}, x_{n}^{k}-x\right\rangle}_{\triangleq A_{1}^{k}}+\underbrace{\sum_{i=1}^{n-1}\left\langle y_{i}^{k}, x_{i}^{k}-x_{n}^{k}\right\rangle}_{\triangleq A_{2}^{k}} .
\end{aligned}
$$

In the above, (a) uses that $y_{i}^{k} \in \partial f_{i}\left(x_{i}^{k}\right)$ and (b) uses (62). We now show that $\alpha_{k} A_{1}^{k}$ and $\alpha_{k} A_{2}^{k}$ both have a finite sum over $k$.

$\alpha_{k} A_{1}^{k}$ is Summable

If $n \in \mathcal{I}_{\mathrm{B}}, A_{1}^{k}$ can be simplified as follows:

$$
\begin{aligned}
& A_{1}^{k} \stackrel{(a)}{=} \frac{\gamma}{\alpha_{k}}\left\langle z^{k}-z^{k+1}, z^{k}-x+\rho_{n}^{k}\left(w_{n}^{k}-y_{n}^{k}\right)+e_{n}^{k}\right\rangle \\
&= \frac{\gamma}{\alpha_{k}}\left\langle z^{k}-z^{k+1}, z^{k}-x\right\rangle+\frac{\gamma \rho_{n}^{k}}{\alpha_{k}}\left\langle z^{k}-z^{k+1}, w_{n}^{k}-y_{n}^{k}\right\rangle \\
& \quad+\frac{\gamma \rho_{n}^{k}}{\alpha_{k}}\left\langle z^{k}-z^{k+1}, e_{n}^{k}\right\rangle \\
& \stackrel{(b)}{\leq} \frac{\gamma}{\alpha_{k}}\left\langle z^{k}-z^{k+1}, z^{k}-x\right\rangle+\frac{\gamma \rho_{n}^{k}}{\alpha_{k}}\left\langle z^{k}-z^{k+1}, w_{n}^{k}-y_{n}^{k}\right\rangle \\
& \quad+\frac{\gamma \rho_{n}^{k}}{2 \alpha_{k}}\left(\left\|z^{k}-z^{k+1}\right\|^{2}+\left\|e_{n}^{k}\right\|^{2}\right) \\
& \stackrel{(c)}{\leq}\left\langle z^{k}-z^{k+1}, z^{k}-x\right\rangle+\frac{\gamma \rho_{n}^{k}}{\alpha_{k}}\left\langle z^{k}-z^{k+1}, w_{n}^{k}-y_{n}^{k}\right\rangle \\
& \quad+\frac{\gamma \bar{\rho}_{n}}{2 \alpha_{k}}\left(\left\|z^{k}-z^{k+1}\right\|^{2}+\delta\left\|z^{k}-x_{n}^{k}\right\|^{2}\right)
\end{aligned}
$$

where (a) uses the proximal update on line 5 of the algorithm for the case $i=n$, (b) used Young's inequality, and (c) uses assumptions 2 and 3. On the other hand, if $n \in \mathcal{I}_{\mathrm{F}}, A_{1}^{k}$ may be written as

$$
A_{1}^{k}=\frac{\gamma}{\alpha_{k}}\left\langle z^{k}-z^{k+1}, z^{k}-x\right\rangle+\frac{\gamma \rho_{n}^{k}}{\alpha_{k}}\left\langle z^{k}-z^{k+1}, w_{n}^{k}-T_{n} z^{k}\right\rangle,
$$

where we have instead used the forward step on line 8, Let $\chi^{k}=w_{n}^{k}-y_{n}^{k}$ when $n \in \mathcal{I}_{\mathrm{B}}$ and $\chi^{k}=w_{n}^{k}-T_{n} z^{k}$ when $n \in \mathcal{I}_{\mathrm{F}}$. Furthermore, let

$$
\theta_{k}=\frac{\gamma \bar{\rho}_{n}}{2 \alpha_{k}}\left(\left\|z^{k}-z^{k+1}\right\|^{2}+\delta\left\|z^{k}-x_{n}^{k}\right\|^{2}\right)
$$

when $n \in \mathcal{I}_{\mathrm{B}}$ and $\theta_{k}=0$ if $n \in \mathcal{I}_{\mathrm{F}}$. Combining (66) and (67), we may write

$$
A_{1}^{k}=\frac{\gamma}{\alpha_{k}}\left\langle z^{k}-z^{k+1}, z^{k}-x\right\rangle+\frac{\gamma \rho_{n}^{k}}{\alpha_{k}}\left\langle z^{k}-z^{k+1}, \chi^{k}\right\rangle+\theta_{k} .
$$


Rewriting the first term in (69) using (5), we obtain

$$
\frac{\gamma}{\alpha_{k}}\left\langle z^{k}-z^{k+1}, z^{k}-x\right\rangle=\frac{\gamma}{2 \alpha_{k}}\left\|z^{k}-z^{k+1}\right\|^{2}+\frac{\gamma}{2 \alpha_{k}}\left(\left\|z^{k}-x\right\|^{2}-\left\|z^{k+1}-x\right\|^{2}\right)
$$

The second term in (69) may be upper bounded using Young's inequality, as follows:

$$
\frac{\gamma \rho_{n}^{k}}{\alpha_{k}}\left\langle z^{k}-z^{k+1}, \chi^{k}\right\rangle \leq \frac{\gamma \rho_{n}^{k}}{2 \alpha_{k}}\left\|z^{k}-z^{k+1}\right\|^{2}+\frac{\gamma \rho_{n}^{k}}{2 \alpha_{k}}\left\|\chi^{k}\right\|^{2}
$$

Thus, we obtain

$$
\alpha_{k} A_{1}^{k} \leq \frac{\gamma}{2}\left(1+\bar{\rho}_{n}\right)\left\|z^{k}-z^{k+1}\right\|^{2}+\frac{\gamma}{2}\left\|z^{k}-x\right\|^{2}-\frac{\gamma}{2}\left\|z^{k+1}-x\right\|^{2}+\frac{\gamma \bar{\rho}_{n}}{2}\left\|\chi^{k}\right\|^{2}+\alpha_{k} \theta_{k} .
$$

Summing (171) over $k$ yields, for $1 \leq k<\bar{K}$,

$$
\sum_{t=1}^{k} \alpha_{t} A_{1}^{t} \leq \frac{\gamma}{2}\left\|z^{1}-x\right\|^{2}+\frac{\gamma}{2}\left(1+\bar{\rho}_{n}\right) \sum_{t=1}^{k}\left\|z^{t}-z^{t+1}\right\|^{2}+\frac{\gamma \bar{\rho}_{n}}{2} \sum_{t=1}^{k}\left\|\chi^{t}\right\|^{2}+\sum_{t=1}^{k} \alpha_{t} \theta_{t} .
$$

We now consider the first three terms on the right-hand side of this relation, employing Lemma 8:

$$
\begin{aligned}
\frac{\gamma}{2}\left\|z^{1}-x\right\|^{2} & \leq \frac{1}{2}\left\|p^{1}-p^{*}\right\|^{2} & & \text { by (17) } \\
\frac{\gamma}{2}\left(1+\bar{\rho}_{n}\right) \sum_{t=1}^{k}\left\|z^{t}-z^{t+1}\right\|^{2} & \leq \frac{1}{2}\left(1+\bar{\rho}_{n}\right) \tau\left\|p^{1}-p^{*}\right\|^{2} & & \text { by (131) } \\
\frac{\gamma \bar{\rho}_{n}}{2} \sum_{t=1}^{k}\left\|\chi^{t}\right\|^{2} & \leq \frac{\gamma \bar{\rho}_{n}}{2} \tau E_{1}\left\|p^{1}-p^{*}\right\|^{2} & & \text { by (134) or (135). }
\end{aligned}
$$

In the last inequality, we use (34) when $n \in \mathcal{I}_{\mathrm{B}}$ and (35) when $n \in \mathcal{I}_{\mathrm{F}}$, and $E_{1}$ is defined in (36). We now consider the last term in (72). When $n \in \mathcal{I}_{\mathrm{B}}$, we have

$$
\begin{array}{rlrl}
\sum_{t=1}^{k} \alpha_{t} \theta_{t} & =\frac{\gamma \bar{\rho}_{n}}{2}\left(\sum_{t=1}^{k}\left\|z^{k}-z^{k+1}\right\|^{2}+\sum_{t=1}^{k} \delta\left\|z^{k}-x_{n}^{k}\right\|^{2}\right) & & \text { by (68) } \\
& \leq \frac{\gamma \bar{\rho}_{n}}{2}\left(\gamma^{-1} \tau\left\|p^{1}-p^{*}\right\|^{2}+\delta \frac{\tau \xi_{1}}{\underline{\beta}^{2} \xi_{2}^{2}}\left\|p^{1}-p^{*}\right\|^{2}\right) & & \text { by (31) and (33) } \\
& =\frac{\tau \bar{\rho}_{n}}{2}\left(1+\frac{\gamma \delta \xi_{1}}{\underline{\beta}^{2} \xi_{2}^{2}}\right)\left\|p^{1}-p^{*}\right\|^{2} . &
\end{array}
$$

The resulting inequality also holds trivially when $n \in \mathcal{I}_{\mathrm{F}}$, since its left-hand side must be zero. Combining all these inequalities, we obtain

$$
\sum_{t=1}^{k} \alpha_{t} A_{1}^{t} \leq \frac{1}{2}\left(1+\left(1+\bar{\rho}_{n}\right) \tau+\gamma \bar{\rho}_{n} \tau E_{1}+\tau \bar{\rho}_{n}\left(1+\frac{\gamma \delta \xi_{1}}{\underline{\beta}^{2} \xi_{2}^{2}}\right)\right)\left\|p^{1}-p^{*}\right\|^{2}
$$




$$
=\frac{1}{2}\left(1+\tau+\bar{\rho}_{n} \tau\left(2+\gamma E_{1}+\frac{\gamma \delta \xi_{1}}{\underline{\beta}^{2} \xi_{2}^{2}}\right)\right)\left\|p^{1}-p^{*}\right\|^{2}
$$

$\underline{\alpha_{k} A_{2}^{k} \text { is Summable }}$

We next perform a similar summability analysis on the second term in (64), $A_{2}^{k}$. We begin by fixing any $1 \leq k<\bar{K}$ and writing

$$
\alpha_{k} A_{2}^{k}=\alpha_{k} \sum_{i=1}^{n-1}\left\langle y_{i}^{k}, x_{i}^{k}-x_{n}^{k}\right\rangle=\alpha_{k} \sum_{i=1}^{n-1}\left\langle w_{i}^{k}, x_{i}^{k}-x_{n}^{k}\right\rangle+\alpha_{k} \sum_{i=1}^{n-1}\left\langle y_{i}^{k}-w_{i}^{k}, x_{i}^{k}-x_{n}^{k}\right\rangle .
$$

Now fix any $i=1, \ldots, n-1$. Using (63),

$$
x_{i}^{k}-x_{n}^{k}=-\frac{1}{\alpha_{k}}\left(w_{i}^{k+1}-w_{i}^{k}\right) .
$$

Substituting this into the first summand in (74) and then using (15), we have

$$
\begin{aligned}
\alpha_{k}\left\langle w_{i}^{k}, x_{i}^{k}-x_{n}^{k}\right\rangle & =\left\langle w_{i}^{k}, w_{i}^{k}-w_{i}^{k+1}\right\rangle \\
= & \left\langle w_{i}^{k}-w_{i}^{*}, w_{i}^{k}-w_{i}^{k+1}\right\rangle+\left\langle w_{i}^{*}, w_{i}^{k}-w_{i}^{k+1}\right\rangle \\
= & \frac{1}{2}\left(\left\|w_{i}^{k}-w_{i}^{*}\right\|^{2}+\left\|w_{i}^{k}-w_{i}^{k+1}\right\|^{2}-\left\|w_{i}^{k+1}-w_{i}^{*}\right\|^{2}\right)+\left\langle w_{i}^{*}, w_{i}^{k}-w_{i}^{k+1}\right\rangle .
\end{aligned}
$$

Using (75) in the second summand in (74) and applying Young's inequality yields

$$
\alpha_{k}\left\langle y_{i}^{k}-w_{i}^{k}, x_{i}^{k}-x_{n}^{k}\right\rangle=\left\langle y_{i}^{k}-w_{i}^{k}, w_{i}^{k}-w_{i}^{k+1}\right\rangle \leq \frac{1}{2}\left\|y_{i}^{k}-w_{i}^{k}\right\|^{2}+\frac{1}{2}\left\|w_{i}^{k}-w_{i}^{k+1}\right\|^{2} .
$$

Substituting (76) and (77) into (74) yields

$$
\begin{aligned}
\alpha_{k} A_{2}^{k} \leq & \frac{1}{2} \sum_{i=1}^{n-1}\left(\left\|w_{i}^{k}-w_{i}^{*}\right\|^{2}+2\left\|w_{i}^{k}-w_{i}^{k+1}\right\|^{2}-\left\|w_{i}^{k+1}-w_{i}^{*}\right\|^{2}+\left\|y_{i}^{k}-w_{i}^{k}\right\|^{2}\right) \\
& +\sum_{i=1}^{n-1}\left\langle w_{i}^{*}, w_{i}^{k}-w_{i}^{k+1}\right\rangle .
\end{aligned}
$$

Summing this relation, we obtain that for any $1 \leq k<\bar{K}$,

$$
\begin{aligned}
\sum_{t=1}^{k} \alpha_{t} A_{2}^{t} \leq & \frac{1}{2} \sum_{i=1}^{n-1}\left(\left\|w_{i}^{1}-w_{i}^{*}\right\|^{2}+2 \sum_{t=1}^{k}\left\|w_{i}^{t}-w_{i}^{t+1}\right\|^{2}+\sum_{t=1}^{k}\left\|y_{i}^{t}-w_{i}^{t}\right\|^{2}\right) \\
& +\sum_{i=1}^{n-1}\left\langle w_{i}^{*}, w_{i}^{1}-w_{i}^{k+1}\right\rangle \\
\leq & \frac{1}{2} \sum_{i=1}^{n-1}\left(\left\|w_{i}^{1}-w_{i}^{*}\right\|^{2}+2 \sum_{t=1}^{k}\left\|w_{i}^{t}-w_{i}^{t+1}\right\|^{2}+\sum_{t=1}^{k}\left\|y_{i}^{t}-w_{i}^{t}\right\|^{2}\right)
\end{aligned}
$$




$$
+\sum_{i=1}^{n-1}\left\|w_{i}^{*}\right\|\left(\left\|w_{i}^{1}-w_{i}^{*}\right\|+\left\|w_{i}^{k+1}-w_{i}^{*}\right\|\right) .
$$

By (32) and (34) from Lemma 8, we then obtain

$$
\sum_{t=1}^{k} \sum_{i=1}^{n-1}\left(\left\|w_{i}^{t}-w_{i}^{t+1}\right\|^{2}+\sum_{t=1}^{k}\left\|y_{i}^{t}-w_{i}^{t}\right\|^{2}\right) \leq \tau\left(1+E_{1}\right)\left\|p^{1}-p^{*}\right\|^{2} .
$$

Finally, we may use (21) to derive

$$
\begin{aligned}
\sum_{i=1}^{n-1}\left\|w_{i}^{*}\right\|\left(\left\|w_{i}^{1}-w_{i}^{*}\right\|+\left\|w_{i}^{k+1}-w_{i}^{*}\right\|\right) & \leq 2\left\|p^{1}-p^{*}\right\| \sum_{i=1}^{n-1}\left\|w_{i}^{*}\right\| \\
& \leq 2 \sqrt{n}\left\|p^{1}-p^{*}\right\|\left\|\mathbf{w}^{*}\right\| \leq 2 \sqrt{n}\left\|p^{1}-p^{*}\right\|\left\|p^{*}\right\|,
\end{aligned}
$$

where in the second inequality we have used Lemma 1. Therefore,

$$
\sum_{t=1}^{k} \alpha_{t} A_{2}^{t} \leq \tau\left(1+E_{1}\right)\left\|p^{1}-p^{*}\right\|^{2}+2 \sqrt{n}\left\|p^{*}\right\|\left\|p^{1}-p^{*}\right\| .
$$

Recalling (61) and using (64) and the fact that $\alpha_{t} \geq \underline{\alpha}$ by Lemma 7, we obtain, for any $1 \leq k<\bar{K}$,

$$
\begin{aligned}
\sum_{i=1}^{n} f_{i}\left(\bar{x}_{i}^{k}\right)-F^{*} & \leq \frac{\sum_{t=1}^{k} \alpha_{t}\left(\sum_{i=1}^{n} f_{i}\left(x_{i}^{t}\right)-F^{*}\right)}{\sum_{t=1}^{k} \alpha_{t}} \\
& \leq \frac{\sum_{t=1}^{k} \alpha_{t}\left(A_{1}^{t}+A_{2}^{t}\right)}{\sum_{t=1}^{k} \alpha_{t}} \\
& \leq \frac{\sum_{t=1}^{k} \alpha_{t}\left(A_{1}^{t}+A_{2}^{t}\right)}{\underline{\alpha} k}=\frac{\xi_{1}}{\beta \xi_{2}} \cdot \frac{\sum_{t=1}^{k} \alpha_{t}\left(A_{1}^{t}+A_{2}^{t}\right)}{k} .
\end{aligned}
$$

Using (73) and (180) in (81) establishes (55) with $E_{2}$ and $E_{3}$ defined in (56) and (157).

$\underline{\text { Part 2: Convergence behavior of } \bar{x}_{i}^{k}-\bar{x}_{l}^{k}}$

Recall from (63) that for all $i=1, \ldots, n-1$,

$$
w_{i}^{t+1}-w_{i}^{t}=\alpha_{t}\left(x_{i}^{t}-x_{n}^{t}\right) .
$$

Summing this equation over $t=1, \ldots, k$ and dividing by $\sum_{t=1}^{k} \alpha_{t}$ yields

$$
(\forall i=1, \ldots, n-1) \quad \bar{x}_{n}^{k}-\bar{x}_{i}^{k}=\frac{w_{i}^{1}-w_{i}^{k+1}}{\sum_{t=1}^{k} \alpha_{t}} .
$$

Therefore, we obtain for all $i, l \in\{1, \ldots, n-1\}$ that

$$
\bar{x}_{l}^{k}-\bar{x}_{i}^{k}=\frac{w_{i}^{1}-w_{l}^{1}-\left(w_{i}^{k+1}-w_{l}^{k+1}\right)}{\sum_{t=1}^{k} \alpha_{t}}
$$




$$
=\frac{w_{i}^{1}-w_{i}^{*}-w_{l}^{1}+w_{l}^{*}-\left(w_{i}^{k+1}-w_{i}^{*}-w_{l}^{k+1}+w_{l}^{*}\right)}{\sum_{t=1}^{k} \alpha_{t}} .
$$

Using (21) and the triangle inequality, we therefore have for all $i, l \in\{1, \ldots, n-1\}$ that

$$
\left\|\bar{x}_{l}^{k}-\bar{x}_{i}^{k}\right\| \leq \frac{\left\|w_{l}^{1}-w_{l}^{*}\right\|+\left\|w_{i}^{1}-w_{i}^{*}\right\|+\left\|w_{l}^{k+1}-w_{l}^{*}\right\|+\left\|w_{i}^{k+1}-w_{i}^{*}\right\|}{\sum_{t=1}^{k} \alpha_{t}} \leq \frac{4\left\|p^{1}-p^{*}\right\|}{\underline{\alpha} k},
$$

where the second inequality uses lemmas 4 and 7 . Finally, we can also use (82) to obtain, for any $i=1, \ldots, n-1$, that

$$
\left\|\bar{x}_{n}^{k}-\bar{x}_{i}^{k}\right\|=\frac{\left\|w_{i}^{1}-w_{i}^{*}-\left(w_{i}^{k}-w_{i}^{*}\right)\right\|}{\sum_{t=1}^{k} \alpha_{t}} \leq \frac{\left.\left\|w_{i}^{1}-w_{i}^{*}\right\|+\| w_{i}^{k}-w_{i}^{*}\right) \|}{\sum_{t=1}^{k} \alpha_{t}} \leq \frac{2\left\|p^{1}-p^{*}\right\|}{\underline{\alpha} k},
$$

where the second inequality again uses lemmas 4 and 7 . Together, (83)-(84) and the definition of $\underline{\alpha}$ imply (58).

Part 3: Convergence rates at a single "splitting" point

We now prove (59) under Assumption 5. We start by establishing that $\bar{x}_{j}^{k}$ is within the domain of each function $f_{i}$ for all $1 \leq k<\bar{K}$ : fix any $1 \leq k<\bar{K}$ and first suppose that $\left|\mathcal{I}_{\mathrm{B}}\right|>0$, which that implies either $j \in \mathcal{I}_{\mathrm{B}} \backslash \mathcal{I}_{\mathrm{L}}$ or $j$ is the unique element of $\mathcal{I}_{\mathrm{B}}$. Since range $\left(\operatorname{prox}_{\rho f_{j}}\right)=\operatorname{dom}\left(f_{j}\right)$, we have that $x_{j}^{k} \in \operatorname{dom}\left(f_{j}\right)$ for all $1 \leq k<\bar{K}$. Since the domain of a convex function is a convex set [2, Proposition 8.2], this implies that $\bar{x}_{j}^{k} \in \operatorname{dom}\left(f_{j}\right)$. The other possibility is that $\left|\mathcal{I}_{\mathrm{B}}\right|=0$, which means that $j \in \mathcal{I}_{\mathrm{F}}$ and therefore $\operatorname{dom}\left(\nabla f_{j}\right)=\mathcal{H}$, implying that $\operatorname{dom}\left(f_{j}\right)=\mathcal{H}$, and thus trivially that $\bar{x}_{j}^{k} \in \operatorname{dom}\left(f_{j}\right)$. Finally recall that for $i \neq j, i \in \mathcal{I}_{\mathrm{L}} \cup \mathcal{I}_{\mathrm{F}}$, therefore either $\operatorname{dom}\left(f_{i}\right)=\mathcal{H}$ or $\left\{x:\|x\| \leq B_{x}\right\} \subseteq \operatorname{dom}\left(f_{i}\right)$. By Lemma 9. $x_{j}^{k} \in\left\{x:\|x\| \leq B_{x}\right\}$ for all $j=1, \ldots, n$ and $1 \leq k<\bar{K}$. Since the ball $B_{x}$ convex, we must also have $\bar{x}_{j}^{k} \in \operatorname{dom}\left(f_{i}\right)$ in this case.

Continuing, we write

$$
\sum_{i=1}^{n} f_{i}\left(\bar{x}_{j}^{k}\right)-F^{*}=\sum_{i=1}^{n} f_{i}\left(\bar{x}_{i}^{k}\right)-F^{*}+\sum_{i \neq j}\left(f_{i}\left(\bar{x}_{j}^{k}\right)-f_{i}\left(\bar{x}_{i}^{k}\right)\right) .
$$

The first summation on the right-hand side of this equation is $\mathrm{O}(1 / k)$ by (55), which has already been established. So we now focus on the terms in second summation.

If $i \in \mathcal{I}_{\mathrm{B}}$ and $i \neq j$, we must have by Assumption 5 that $i \in \mathcal{I}_{\mathrm{L}}$. Therefore,

$$
f_{i}\left(\bar{x}_{j}^{k}\right)-f_{i}\left(\bar{x}_{i}^{k}\right) \leq M_{i}\left\|\bar{x}_{i}^{k}-\bar{x}_{j}^{k}\right\| \leq \frac{4 M_{i}\left\|p^{1}-p^{*}\right\|}{\underline{\alpha} k}
$$

where the the second inequality arises from (58).

Otherwise, we have $i \in \mathcal{I}_{\mathrm{F}}$ and $i \neq j$, and since $\operatorname{dom}\left(\nabla f_{i}\right)=\mathcal{H}$, we may use the subgradient inequality to write

$$
\begin{aligned}
f_{i}\left(\bar{x}_{j}^{k}\right)-f_{i}\left(\bar{x}_{i}^{k}\right) & \leq\left\langle\nabla f_{i}\left(\bar{x}_{j}^{k}\right), \bar{x}_{j}^{k}-\bar{x}_{i}^{k}\right\rangle \\
& =\left\langle\nabla f_{i}\left(x_{i}^{k}\right), \bar{x}_{j}^{k}-\bar{x}_{i}^{k}\right\rangle+\left\langle\nabla f_{i}\left(\bar{x}_{j}^{k}\right)-\nabla f_{i}\left(x_{i}^{k}\right), \bar{x}_{j}^{k}-\bar{x}_{i}^{k}\right\rangle
\end{aligned}
$$




$$
\begin{aligned}
& \leq\left\langle y_{i}^{k}, \bar{x}_{j}^{k}-\bar{x}_{i}^{k}\right\rangle+L_{i}\left\|\bar{x}_{j}^{k}-x_{i}^{k}\right\|\left\|\bar{x}_{j}^{k}-\bar{x}_{i}^{k}\right\| \\
& \leq\left\|y_{i}^{k}\right\|\left\|\bar{x}_{j}^{k}-\bar{x}_{i}^{k}\right\|+L_{i}\left(\left\|\bar{x}_{j}^{k}\right\|+\left\|x_{i}^{k}\right\|\right)\left\|\bar{x}_{j}^{k}-\bar{x}_{i}^{k}\right\| \\
& =\left(\left\|y_{i}^{k}\right\|+L_{i}\left(\left\|\bar{x}_{j}^{k}\right\|+\left\|\bar{x}_{i}^{k}\right\|\right)\right)\left\|\bar{x}_{j}^{k}-\bar{x}_{i}^{k}\right\|,
\end{aligned}
$$

where the second inequality uses that $y_{i}^{k}=\nabla f_{i}\left(x_{i}^{k}\right)$ for $i \in \mathcal{I}_{\mathrm{F}}$, the Cauchy-Schwarz inequality, and the Lipschitz continuity of $\nabla f_{i}$. Lemma 9 assures us that $\left\|x_{i}^{k}\right\|,\left\|\bar{x}_{j}^{k}\right\| \leq B_{x}$ and $\left\|y_{i}^{k}\right\| \leq B_{y}$, which in conjunction with (58) and $L_{i} \leq \bar{L}$ leads to

$$
f_{i}\left(\bar{x}_{j}^{k}\right)-f_{i}\left(\bar{x}_{i}^{k}\right) \leq \frac{4\left(B_{y}+2 \bar{L} B_{x}\right)\left\|p^{1}-p^{*}\right\|}{\underline{\alpha} k} .
$$

Substituting (55), (86), and (88) into (85), we obtain

$$
\begin{aligned}
& \sum_{i=1}^{n} f_{i}\left(\bar{x}_{j}^{k}\right)-F^{*}=\sum_{i=1}^{n} f_{i}\left(\bar{x}_{i}^{k}\right)-F^{*}+\sum_{i \neq j}\left(f_{i}\left(\bar{x}_{j}^{k}\right)-f_{i}\left(\bar{x}_{i}^{k}\right)\right) \\
& \leq \frac{E_{2}\left\|p^{1}-p^{*}\right\|^{2}+E_{3}\left\|p^{1}-p^{*}\right\|}{k}+\sum_{i \in \mathcal{I}_{\mathrm{L}}} \frac{4 M_{i}\left\|p^{1}-p^{*}\right\|}{\underline{\alpha} k}+\frac{4\left|\mathcal{I}_{\mathrm{F}}\right| B_{y}\left(1+2 \bar{L} B_{x}\right)\left\|p^{1}-p^{*}\right\|}{\underline{\alpha} k} \\
& \leq \frac{E_{2}\left\|p^{1}-p^{*}\right\|^{2}+E_{3}\left\|p^{1}-p^{*}\right\|}{k}+\frac{4\left\|p^{1}-p^{*}\right\|}{\underline{\alpha} k}\left(\sum_{i \in \mathcal{I}_{\mathrm{L}}} M_{i}+n\left(B_{y}+2 \bar{L} B_{x}\right)\right) .
\end{aligned}
$$

This establishes (59) with $E_{4}$ defined in (600).

\section{Consequences of Strong Monotonicity}

We now investigate the consequences of strong monotonicity in (11) for the convergence rate of projective splitting.

Assumption 6. For (11), there is some $l \in\{1, \ldots, n\}$ for which $T_{l}$ is strongly monotone.

Note that under Assumption 6 it is immediate that the solution of (1) is unique.

Theorem 2. Suppose assumptions 1, 2, 0, and 6 hold. Let $z^{*}$ be the unique solution to (1). Fix $p^{*}=\left(z^{*}, \mathbf{w}^{*}\right) \in \mathcal{S}$. If $\bar{K}=+\infty$ then $x_{i}^{k} \rightarrow z^{*}$ for $i=1, \ldots, n$ and $z^{k} \rightarrow z^{*}$. If $\bar{K}<\infty$, $x_{i}^{\bar{K}}=z^{\bar{K}+1}=z^{*}$ for $i=1, \ldots, n$. Furthermore for all $1 \leq k<\bar{K}$

$$
\left\|x_{\mathrm{avg}}^{k}-z^{*}\right\|^{2} \leq \frac{\xi_{1}\left\|p^{1}-p^{*}\right\|^{2}}{\underline{\beta} \xi_{2} \mu k}
$$

where $x_{\mathrm{avg}}^{k}=\frac{1}{k} \sum_{i=1}^{t} x_{l}^{i}$ and $\xi_{1}$ and $\xi_{2}$ are defined in (22) and (28) respectively.

Proof. Regarding the case $\bar{K}<\infty$, optimality of $x_{i}^{\bar{K}}$ and $z^{\bar{K}+1}$ was shown in [15, Lemma 5].

Let $w_{n}^{*}=-\sum_{i=1}^{n-1} w_{i}^{*}$. Using this notation and recalling the affine function defined in (8), we may write

$$
-\varphi_{k}\left(z^{*}, \mathbf{w}^{*}\right)=\sum_{i=1}^{n}\left\langle z^{*}-x_{i}^{k}, w_{i}^{*}-y_{i}^{k}\right\rangle
$$




$$
\begin{aligned}
& =\sum_{i \neq l}\left\langle z^{*}-x_{i}^{k}, w_{i}^{*}-y_{i}^{k}\right\rangle+\left\langle z^{*}-x_{l}^{k}, w_{l}^{*}-y_{l}^{k}\right\rangle \\
& \geq \mu\left\|z^{*}-x_{l}^{k}\right\|^{2},
\end{aligned}
$$

where the inequality uses that $\left(x_{i}^{k}, y_{i}^{k}\right)$ and $\left(z^{*}, w_{i}^{*}\right)$ are in gra $T_{i}$, along with the monotonicity of $T_{i}$ for $i \neq l$, and also the strong monotonicity of $T_{l}$. Now, Lemma 6 implies that $\varphi_{k}\left(z^{k}, \mathbf{w}^{k}\right) \geq 0$. Therefore, for any $1 \leq k<\bar{K}$,

$$
\begin{aligned}
\mu\left\|z^{*}-x_{l}^{k}\right\|^{2} & \leq \varphi_{k}\left(z^{k}, \mathbf{w}^{k}\right)-\varphi_{k}\left(z^{*}, \mathbf{w}^{*}\right) \\
& \stackrel{(a)}{=}\left\langle\nabla \varphi_{k}, p^{k}-p^{*}\right\rangle \\
& \stackrel{(b)}{=} \frac{1}{\alpha_{k}}\left\langle p^{k}-p^{k+1}, p^{k}-p^{*}\right\rangle \\
& \stackrel{(c)}{=} \frac{1}{2 \alpha_{k}}\left(\left\|p^{k}-p^{k+1}\right\|^{2}+\left\|p^{k}-p^{*}\right\|^{2}-\left\|p^{k+1}-p^{*}\right\|^{2}\right) \\
& \stackrel{(d)}{\leq} \frac{1}{2 \underline{\alpha}}\left(\left\|p^{k}-p^{k+1}\right\|^{2}+\left\|p^{k}-p^{*}\right\|^{2}-\left\|p^{k+1}-p^{*}\right\|^{2}\right) .
\end{aligned}
$$

Above, (a) uses that $\varphi_{k}$ is affine, (b) employs (17) from Lemma 4, (c) uses (5), and (d) uses Lemma 7 and (18) from Lemma 4. Summing the resulting inequality yields, for all $1 \leq k<\bar{K}$

$$
\mu \sum_{t=1}^{k}\left\|z^{*}-x_{l}^{t}\right\|^{2} \leq \frac{1}{2 \underline{\alpha}}\left(\sum_{t=1}^{k}\left\|p^{t}-p^{t+1}\right\|^{2}+\left\|p^{1}-p^{*}\right\|^{2}\right) \leq \frac{\xi_{1}}{\underline{\beta} \xi_{2}}\left\|p^{1}-p^{*}\right\|^{2},
$$

where the second inequality uses Lemma 4 and (19) from Lemma 7. So, when $\bar{K}=\infty$, we have $x_{l}^{k} \rightarrow z^{*}$. Lemma 8 asserts that $\nabla \varphi_{k} \rightarrow 0$ in the $\bar{K}=\infty$ case, so from (9) we conclude that $x_{i}^{k} \rightarrow z^{*}$ for $i=1, \ldots, n$. Lemma 8 also establishes that $\varphi_{k}\left(p^{k}\right) \rightarrow 0$, so by Lemma 6 , we have $\left\|z^{k}-x_{i}^{k}\right\| \rightarrow 0$ for all $i=1, \ldots, n$ and hence $z^{k} \rightarrow z^{*}$. Finally, the convexity of the function $\|\cdot\|^{2}$ in conjunction with (91) yields (89).

\section{Linear Convergence Under Strong Monotonicity and Cocoercivity}

If we introduce a cocoercivity assumption along with strong monotonicity, we can derive linear convergence. We require that all but one of the operators be cocoercive. Without loss of generality, we designate $T_{n}$ to be be the operator that need not be cocoercive.

Assumption 7. In (1), suppose that for $i=1, \ldots, n-1$, the operator $T_{i}$ is cocoercive with parameter $\Gamma_{i}>0$. Let $\bar{\Gamma}=\max _{i=1, \ldots, n-1} \Gamma_{i}$.

If this assumption holds, then $T_{1}, \ldots, T_{n-1}$ are all single-valued. If Assumption 6 also holds, this single-valuedness implies that not only is the solution $z^{*}$ to (1) unique, but that the extended solution set $\mathcal{S}$ must be singleton of the form $\left\{\left(z^{*}, w_{1}^{*}, \ldots, w_{n-1}^{*}\right)\right\}=$ $\left\{\left(z^{*}, T_{1} z^{*}, \ldots, T_{n-1} z^{*}\right)\right\}$. 
Theorem 3. Suppose assumptions 1, 2, [, 6, and 7 hold. Let $z^{*}$ be the unique solution to (1) and take $p^{*}=\left(z^{*}, \mathbf{w}^{*}\right)=\left(z^{*}, T_{1} z^{*}, \ldots, T_{n-1} z^{*}\right) \in \mathcal{S}$. If $\bar{K}<\infty, p^{\bar{K}+1}=p^{*}$. For all $1 \leq k<\bar{K}$,

$$
\left\|p^{k+1}-p^{*}\right\|^{2} \leq\left(1-E_{5}\right)\left\|p^{k}-p^{*}\right\|^{2}
$$

where

$$
\left.\left.E_{5}=\frac{1}{2}\left(\frac{8 \xi_{1}^{2}(1+\gamma)^{2} \max \left\{\mu^{-1}, \bar{\Gamma}\right\}^{2}+2 \gamma \xi_{1}}{\underline{\beta}^{2} \xi_{2}^{2}}+2 E_{1}\right)^{-1} \in\right] 0,1 / 4\right] .
$$

Proof. For the finite-termination case, optimality of $p^{\bar{K}+1}$ was established in [15, Lemma 5]. Henceforth, we thus assume that $\bar{K}=\infty$. The key idea of the proof is to show that for $E_{5}$ defined in (93),

$$
E_{5}\left\|p^{k}-p^{*}\right\|^{2} \leq\left\|p^{k+1}-p^{k}\right\|^{2},
$$

which, when used with (18) of Lemma 4, implies

$$
\left\|p^{k+1}-p^{*}\right\|^{2} \leq\left\|p^{k}-p^{*}\right\|^{2}-\left\|p^{k+1}-p^{k}\right\|^{2} \leq\left(1-E_{5}\right)\left\|p^{k}-p^{*}\right\|^{2} .
$$

We now establish (94). For $1 \leq k<\bar{K}$, we have $\varphi_{k}\left(p^{k}\right) \geq 0$ and hence

$$
\begin{aligned}
\varphi_{k}\left(p^{k}\right)-\varphi_{k}\left(p^{*}\right) & \geq \sum_{i=1}^{n}\left\langle z^{*}-x_{i}^{k}, w_{i}^{*}-y_{i}^{k}\right\rangle \\
& =\frac{1}{2} \sum_{i=1}^{n}\left\langle z^{*}-x_{i}^{k}, w_{i}^{*}-y_{i}^{k}\right\rangle+\frac{1}{2} \sum_{i=1}^{n}\left\langle z^{*}-x_{i}^{k}, w_{i}^{*}-y_{i}^{k}\right\rangle \\
& \geq \frac{\mu}{2}\left\|z^{*}-x_{l}^{k}\right\|^{2}+\sum_{i=1}^{n-1} \frac{1}{2 \Gamma_{i}}\left\|y_{i}^{k}-w_{i}^{*}\right\|^{2} .
\end{aligned}
$$

The last inequality here follows because $\sum_{i=1}^{n}\left\langle z^{*}-x_{i}^{k}, w_{i}^{*}-y_{i}^{k}\right\rangle \geq \mu\left\|z^{*}-x_{l}^{k}\right\|^{2}$ by the same logic as in (90), and by the cocoerciveness of $T_{1}, \ldots, T_{n-1}$. We therefore obtain

$$
\begin{aligned}
\frac{\mu}{2}\left\|z^{*}-x_{l}^{k}\right\|^{2}+\sum_{i=1}^{n-1} \frac{1}{2 \Gamma_{i}}\left\|y_{i}^{k}-w_{i}^{*}\right\|^{2} \leq \varphi_{k}\left(p^{k}\right)-\varphi_{k}\left(p^{*}\right) & =\left\langle\nabla \varphi_{k}, p^{k}-p^{*}\right\rangle \\
& =\frac{1}{\alpha_{k}}\left\langle p^{k}-p^{k+1}, p^{k}-p^{*}\right\rangle,
\end{aligned}
$$

where we have again used that $\varphi_{k}$ is affine, and also (17). Continuing, we use Young's inequality applied to (95) to write, for any $\nu>0$,

$$
\frac{\mu}{2}\left\|z^{*}-x_{l}^{k}\right\|^{2}+\sum_{i=1}^{n-1} \frac{1}{2 \Gamma_{i}}\left\|y_{i}^{k}-w_{i}^{*}\right\|^{2} \leq \frac{1}{2 \nu \underline{\alpha}^{2}}\left\|p^{k+1}-p^{k}\right\|^{2}+\frac{\nu}{2}\left\|p^{k}-p^{*}\right\|^{2},
$$


which implies

$$
\left\|z^{*}-x_{l}^{k}\right\|^{2}+\sum_{i=1}^{n-1}\left\|y_{i}^{k}-w_{i}^{*}\right\|^{2} \leq \frac{\xi_{5}}{\nu \underline{\alpha}^{2}}\left\|p^{k+1}-p^{k}\right\|^{2}+\xi_{5} \nu\left\|p^{k}-p^{*}\right\|^{2},
$$

where $\xi_{5}=\max \left\{\mu^{-1}, \bar{\Gamma}\right\}$. From Lemma 1, we then deduce that

$$
\begin{aligned}
& \left\|p^{k}-p^{*}\right\|^{2}=\gamma\left\|z^{k}-z^{*}\right\|^{2}+\sum_{i=1}^{n-1}\left\|w_{i}^{k}-w_{i}^{*}\right\|^{2} \\
& \leq 2 \gamma\left\|z^{*}-x_{l}^{k}\right\|^{2}+2 \gamma\left\|x_{l}^{k}-z^{k}\right\|^{2} \\
& \quad+2 \sum_{i=1}^{n-1}\left\|y_{i}^{k}-w_{i}^{*}\right\|^{2}+2 \sum_{i=1}^{n-1}\left\|y_{i}^{k}-w_{i}^{k}\right\|^{2} . \\
& \leq 2(1+\gamma)\left(\left\|z^{*}-x_{l}^{k}\right\|^{2}+\sum_{i=1}^{n-1}\left\|y_{i}^{k}-w_{i}^{*}\right\|^{2}\right)+2 \gamma\left\|x_{l}^{k}-z^{k}\right\|^{2}+2 \sum_{i=1}^{n-1}\left\|y_{i}^{k}-w_{i}^{k}\right\|^{2} .
\end{aligned}
$$

Substituting the upper bound (96) for the term in parentheses in (97), and then using (33) and (34) from Lemma 8 on the other two terms, we conclude that

$$
\begin{gathered}
\left\|p^{k}-p^{*}\right\|^{2} \leq 2(1+\gamma)\left(\frac{\xi_{5}}{\nu \underline{\alpha}^{2}}\left\|p^{k+1}-p^{k}\right\|^{2}+\xi_{5} \nu\left\|p^{k}-p^{*}\right\|^{2}\right)+\frac{2 \gamma \xi_{1}}{\underline{\beta}^{2} \xi_{2}^{2}}\left\|p^{k+1}-p^{k}\right\|^{2} \\
+2 E_{1}\left\|p^{k+1}-p^{k}\right\|^{2} .
\end{gathered}
$$

Rearranging this inequality yields

$$
\left(1-2 \nu(1+\gamma) \xi_{5}\right)\left\|p^{k}-p^{*}\right\|^{2} \leq\left(\frac{2(1+\gamma) \xi_{5}}{\nu \underline{\alpha}^{2}}+\frac{2 \gamma \xi_{1}}{\underline{\beta}^{2} \xi_{2}^{2}}+2 E_{1}\right)\left\|p^{k+1}-p^{k}\right\|^{2} .
$$

We now set

$$
\nu=\frac{1}{4(1+\gamma) \xi_{5}}
$$

Using this value of $\nu$ in (98) implies (94) with

$$
E_{5}=\frac{1}{2}\left(\frac{8(1+\gamma)^{2}\left(\xi_{5}\right)^{2}}{\underline{\alpha}^{2}}+\frac{2 \gamma \xi_{1}}{\underline{\beta}^{2} \xi_{2}^{2}}+2 E_{1}\right)^{-1}
$$

which reduces to the expression in (93). Considering (93), we note that since $\xi_{2}>0, \underline{\beta}>0$, $\mu>0$, and $E_{1}<\infty$, we must have $E_{5}>0$.

Finally, we show that $E_{5} \leq 1 / 4$ as claimed in (93) (in particular, this precludes the nonsensical situation that $E_{5}>1$ ). We write

$$
E_{5}=\frac{1}{2}\left(\frac{8 \xi_{1}^{2}(1+\gamma)^{2} \max \left\{\mu^{-1}, \bar{\Gamma}\right\}^{2}+2 \gamma \xi_{1}}{\underline{\beta}^{2} \xi_{2}^{2}}+2 E_{1}\right)^{-1}
$$




$$
\begin{aligned}
& \stackrel{(a)}{\leq} \frac{\xi_{2}^{2}}{\gamma \xi_{1}} \\
& =\frac{\min \left\{(1-\sigma) \bar{\rho}^{-1}, \min _{j \in \mathcal{I}_{\mathrm{F}}}\left\{\bar{\rho}_{j}^{-1}-L_{j}\right\}\right\}^{2}}{2 n \gamma\left[1+2 \gamma^{-1}\left(\bar{L}^{2}\left|\mathcal{I}_{\mathrm{F}}\right|+\underline{\rho}^{-2}(1+\delta)\right)\right]} \\
& \leq \frac{\min \left\{(1-\sigma) \bar{\rho}^{-1}, \min _{j \in \mathcal{I}_{\mathrm{F}}}\left\{\bar{\rho}_{j}^{-1}-L_{j}\right\}\right\}^{2}}{4 n \underline{\rho}^{-2}(1+\delta)} \\
& \stackrel{(b)}{\leq} \frac{\left((1-\sigma) \bar{\rho}^{-1}+\sum_{j \in \mathcal{I}_{\mathrm{F}}}\left(\bar{\rho}_{j}^{-1}-L_{j}\right)\right)^{2}}{4 n \underline{\rho}^{-2}(1+\delta)\left(\left|\mathcal{I}_{\mathrm{F}}\right|+1\right)^{2}} \\
& \stackrel{(c)}{\leq} \frac{\bar{\rho}^{-2}+\sum_{j \in \mathcal{I}_{\mathrm{F}}} \bar{\rho}_{j}^{-2}}{4 n \underline{\rho}^{-2}(1+\delta)\left(\left|\mathcal{I}_{\mathrm{F}}\right|+1\right)} \stackrel{(d)}{\leq} \frac{1}{4 n(1+\delta)} \leq \frac{1}{4},
\end{aligned}
$$

where we employ the following reasoning: first, (a) uses that all terms within the parentheses in (100) are positive and that $\underline{\beta}<2$. In (b), we use that the minimum of a set of numbers cannot exceed its average. Inequality (c) follows by observing that $(1-\sigma) \leq 1$ and $-L_{j} \leq 0$, and then using Lemma 1, Finally, (d) uses that $\underline{\rho} \leq \bar{\rho}_{j}$ and $\underline{\rho} \leq \bar{\rho}$.

\section{$9 \quad$ Simplifications when $n=1$}

Suppose $n=1$, in which case we have either $1 \in \mathcal{I}_{\mathrm{B}}$ or $1 \in \mathcal{I}_{\mathrm{F}}$. In either case, using the convention discussed at the beginning of Section 2.1, we have $w_{1}^{k}=0$ for all $k$ and the affine function defined in (8) becomes

$$
\varphi_{k}(p)=\varphi_{k}(z)=\left\langle z-x^{k}, y^{k}\right\rangle
$$

where we have written $x_{1}^{k}=x^{k}$ and $y_{1}^{k}=y^{k}$.

If $1 \in \mathcal{I}_{\mathrm{B}}$, using $w_{1}^{k}=0$, in the update on lines 4,6 of the algorithm yields

$$
x^{k}+\rho^{k} y^{k}=z^{k} \quad \text { and } \quad y^{k} \in T x^{k},
$$

where we have written $T_{1}=T$ and $\rho_{1}^{k}=\rho^{k}$. This implies that $\varphi_{k}\left(z^{k}\right)=\rho^{k}\left\|y^{k}\right\|^{2}$. Furthermore, $\nabla_{z} \varphi_{k}=\gamma^{-1} y^{k}$ and so $\left\|\nabla_{z} \varphi_{k}\right\|^{2}=\gamma \cdot \gamma^{-2}\left\|y^{k}\right\|^{2}=\gamma^{-1}\left\|y^{k}\right\|^{2}$. Therefore, using (17), we have

$$
\begin{aligned}
z^{k+1}=z^{k}-\frac{\beta_{k} \varphi_{k}\left(p^{k}\right)}{\left\|\nabla \varphi_{k}\right\|^{2}} \nabla_{z} \varphi_{k} & =z^{k}-\beta_{k} \rho^{k} y^{k} \\
& =\left(1-\beta_{k}\right) z^{k}+\beta_{k} x^{k}=\left(1-\beta_{k}\right) z^{k}+\beta_{k} \operatorname{prox}_{\rho^{k}}\left(z^{k}\right) .
\end{aligned}
$$

Thus, when $n=1$ and $1 \in \mathcal{I}_{\mathrm{B}}$, projective splitting reduces to the relaxed proximal point method of [9]; see also [2, Theorem 23.41]. In fact, when one allows for approximate evaluation of resolvents, projective splitting with $n=1 \in \mathcal{I}_{\mathrm{B}}$ reduces to the hybrid projection proximal-point method of Solodov and Svaiter [23, Algorithm 1.1]. However, the error criterion of [23, Eq. (1.1)] is more restrictive than the conditions (11) $-(12)$ that we propose in Assumption 2 
On the other hand, if $1 \in \mathcal{I}_{\mathrm{F}}$, considering lines 8 . 0 or the algorithm with $w_{1}^{k}=0$ yields

$$
x^{k}=z^{k}-\rho^{k} T z^{k} \quad \text { and } \quad y^{k}=T x^{k} .
$$

Furthermore, we have

$$
\varphi_{k}\left(z^{k}\right)=\rho^{k}\left\langle T z^{k}, T x^{k}\right\rangle \quad \text { and } \quad \nabla_{z} \varphi_{k}=\gamma^{-1} T x^{k} .
$$

Therefore,

$$
z^{k+1}=z^{k}-\frac{\beta_{k} \varphi_{k}\left(p^{k}\right)}{\left\|\nabla \varphi_{k}\right\|^{2}} \nabla_{z} \varphi_{k}=z^{k}-\frac{\beta_{k} \rho^{k}\left\langle T z^{k}, T x^{k}\right\rangle}{\left\|T x^{k}\right\|^{2}} T x^{k} .
$$

Thus, the method reduces to

$$
\begin{aligned}
x^{k} & =z^{k}-\rho^{k} T z^{k} \\
z^{k+1} & =z^{k}-\tilde{\rho}^{k} T x^{k},
\end{aligned}
$$

where

$$
\tilde{\rho}^{k}=\frac{\beta_{k} \rho^{k}\left\langle T z^{k}, T x^{k}\right\rangle}{\left\|T x^{k}\right\|^{2}} .
$$

This is the unconstrained version of the extragradient method [16]. When $\beta_{k}=1$, the stepsize (101) corresponds to the extragradient stepsize proposed by Iusem and Svaiter in [14]. Furthermore, the backtracking linesearch for the extragradient method also proposed in [14] is almost equivalent in the unconstrained case to the linesearch we proposed for processing individual operators within projective splitting in [15], except that Iusem and Svaiter use a more restrictive termination condition (perhaps necessary because they also account for the constrained case of the extragradient method).

While these observations may be of interest in their own right, they also have implications for the convergence rate analysis. In particular, that projective splitting reduces to the proximal point method suggests that the $\mathrm{O}(1 / k)$ ergodic convergence rate for (52) derived in Section 6 cannot be improved beyond a constant factor. This is because the same rate is unimprovable for the proximal point method under the assumption that the stepsize is bounded from above and below [12.

\section{References}

[1] Alotaibi, A., Combettes, P.L., Shahzad, N.: Solving Coupled Composite Monotone Inclusions by Successive Fejér Approximations of their Kuhn-Tucker Set. SIAM Journal on Optimization 24(4), 2076-2095 (2014)

[2] Bauschke, H.H., Combettes, P.L.: Convex analysis and monotone operator theory in Hilbert spaces, 2nd edn. Springer (2017)

[3] Boyd, S., Parikh, N., Chu, E., Peleato, B., Eckstein, J.: Distributed optimization and statistical learning via the alternating direction method of multipliers. Foundations and Trends in Machine Learning 3(1), 1-122 (2011) 
[4] Combettes, P.L., Eckstein, J.: Asynchronous block-iterative primal-dual decomposition methods for monotone inclusions. Mathematical Programming 168(1-2), 645-672 (2018)

[5] Combettes, P.L., Pesquet, J.C.: Proximal splitting methods in signal processing. In: Fixed-point algorithms for inverse problems in science and engineering, pp. 185-212. Springer (2011)

[6] Combettes, P.L., Wajs, V.R.: Signal recovery by proximal forward-backward splitting. Multiscale Modeling \& Simulation 4(4), 1168-1200 (2005)

[7] Davis, D., Yin, W.: A three-operator splitting scheme and its optimization applications. Set-Valued and Variational Analysis 25(4), 829-858 (2017)

[8] Eckstein, J.: A simplified form of block-iterative operator splitting and an asynchronous algorithm resembling the multi-block alternating direction method of multipliers. Journal of Optimization Theory and Applications 173(1), 155-182 (2017)

[9] Eckstein, J., Bertsekas, D.P.: On the Douglas-Rachford splitting method and the proximal point algorithm for maximal monotone operators. Math. Programming 55(3), 293-318 (1992)

[10] Eckstein, J., Svaiter, B.F.: A family of projective splitting methods for the sum of two maximal monotone operators. Mathematical Programming 111(1), 173-199 (2008)

[11] Eckstein, J., Svaiter, B.F.: General projective splitting methods for sums of maximal monotone operators. SIAM Journal on Control and Optimization 48(2), 787-811 (2009)

[12] Güler, O.: On the convergence of the proximal point algorithm for convex minimization. SIAM Journal on Control and Optimization 29(2), 403-419 (1991)

[13] Haugazeau, Y.: Sur les inéquations variationnelles et la minimisation de fonctionnelles convexes. Ph.D. thesis, Université de Paris, Paris (1968)

[14] Iusem, A., Svaiter, B.: A variant of Korpelevich's method for variational inequalities with a new search strategy. Optimization 42(4), 309-321 (1997)

[15] Johnstone, P.R., Eckstein, J.: Projective splitting with forward steps: Asynchronous and block-iterative operator splitting. arXiv preprint arXiv:1803.07043 (2018)

[16] Korpelevich, G.: Extragradient method for finding saddle points and other problems. Matekon 13(4), 35-49 (1977)

[17] Lions, P.L., Mercier, B.: Splitting algorithms for the sum of two nonlinear operators. SIAM Journal on Numerical Analysis 16(6), 964-979 (1979)

[18] Machado, M.P.: Projective method of multipliers for linearly constrained convex minimization. arXiv preprint arXiv:1609.00467 (2016) 
[19] Machado, M.P.: On the complexity of the projective splitting and Spingarn's methods for the sum of two maximal monotone operators. arXiv preprint arXiv:1707.08655 (2017)

[20] Mercier, B., Vijayasundaram, G.: Lectures on topics in finite element solution of elliptic problems. Tata Institute of Fundamental Research, Bombay (1979)

[21] Nguyen, T.P., Pauwels, E., Richard, E., Suter, B.W.: Extragradient method in optimization: Convergence and complexity. Journal of Optimization Theory and Applications 176(1), 137-162 (2018)

[22] Rockafellar, R.T.: Monotone operators and the proximal point algorithm. SIAM J. Control Optimization 14(5), 877-898 (1976)

[23] Solodov, M.V., Svaiter, B.F.: A hybrid projection-proximal point algorithm. Journal of convex analysis 6(1), 59-70 (1999)

[24] Tseng, P.: A modified forward-backward splitting method for maximal monotone mappings. SIAM Journal on Control and Optimization 38(2), 431-446 (2000) 